JOURNAL OF

SYMPLECTIC GEOMETRY

Volume 8, Number 3, 267-298, 2010

\title{
COMPACTNESS FOR HOLOMORPHIC CURVES WITH SWITCHING LAGRANGIAN BOUNDARY CONDITIONS
}

\author{
K. Cieliebak, T. Ekholm, and J. Latschev
}

\begin{abstract}
We prove a compactness result for holomorphic curves with boundary on an immersed Lagrangian submanifold with clean self-intersection. As an important consequence, we show that the number of intersections of such holomorphic curves with the self-intersection locus is uniformly bounded in terms of the Hofer energy.
\end{abstract}

\section{Introduction}

In this paper we prove a compactness result for holomorphic curves with boundary on an immersed Lagrangian submanifold with clean selfintersection along a compact submanifold $K$. In contrast with other compactness results for curves with Lagrangian boundary conditions of which we are aware, we do not a priori restrict the number of times the boundary of the curve "switches branches" at $K$. In fact, we derive as an important consequence of the compactness theorem that the number of intersections of such holomorphic curves with $K$, and hence the number of "switches," is uniformly bounded in terms of the Hofer energy. This finiteness result is an essential ingredient in the proof in [5] of the isomorphism of degree 0 Legendrian contact homology of the unit conormal bundle of a knot $K \subset \mathbb{R}^{3}$ with the cord algebra defined in $[\mathbf{1 6}]$.

Consider a symplectic manifold $(X, \omega)$ and an immersed Lagrangian submanifold $L \subset X$ with clean self-intersection along a compact submanifold $K$. Let $J$ be an $\omega$-compatible almost complex structure on $X$. We assume that near $K$ the structure $J$ is integrable and $L$ is real analytic. Let $(S, j)$ be a connected Riemmann surface with boundary $\partial S$. A holomorphic curve $f:(S, \partial S, j) \rightarrow(X, L, J)$ is a continuous map $f: S \rightarrow X$ which maps $\partial S$ to $L$ and is $(j, J)$-holomorphic in the interior. We allow $(X, L, \omega, J)$ to be noncompact with cylindrical ends as in [9], and $S$ to have punctures in the interior as well as on the boundary (see Section 3 for the precise setup). However, we do not treat intersections of $\left.f\right|_{\partial S}$ with $K$ - which we call switches 
- as boundary punctures. In particular, we do not impose any constraints on the number and types of switches.

Our first result states that the compactness result in symplectic field theory $[3,7]$ carries over to this setting. See Section 4 for the precise statement.

Theorem 1.1. Under suitable hypotheses on $(X, L, \omega, J)$ each sequence of holomorphic curves $f_{n}:\left(S_{n}, \partial S_{n}, j_{n}\right) \rightarrow(X, L, J)$ of fixed signature and uniformly bounded energy has a subsequence converging in the sense of $[\mathbf{3}]$ to a stable holomorphic curve.

As a consequence, we obtain the following finiteness result for the number of switches, which was the primary motivation for this work.

Theorem 1.2. In the situation of Theorem 1.1, suppose in addition that $(X, L, \omega=d \lambda)$ is exact with convex end. Then for each $s \in \mathbb{N}$ and $C>0$ there exists a constant $\kappa(s, C) \in \mathbb{N}$ such that every holomorphic disc $f$ : $(\dot{D}, \partial \dot{D}, j) \rightarrow(X, L, J)$ with at most $s$ boundary punctures and energy $\leq C$ has at most $\kappa(s, C)$ switches.

The case $s=1$ of this finiteness result is an essential ingredient in the proof in [5] of the isomorphism of degree 0 Legendrian contact homology of the unit conormal bundle of a knot $K \subset \mathbb{R}^{3}$ with the cord algebra defined in [16]. This isomorphism is constructed by counting one-punctured holomorphic discs in $T^{*} \mathbb{R}^{3}$ with boundary on the immersed Lagrangian submanifold $L=N K \cup \mathbb{R}^{3}$, where the conormal bundle $N K \subset T^{*} \mathbb{R}^{3}$ of $K$ and the zero section $\mathbb{R}^{3}$ intersect cleanly along the knot $K$.

Holomorphic discs with boundary on cleanly intersecting Lagrangian submanifolds are also studied in $[\mathbf{2}]$, where what we call a switch is called a jump. Besides being in the somewhat different setting of Hamiltonian Floer theory, the compactness discussion in [2, section 6.1] assumes a fixed number of switches, and so it does not yield any result similar to Theorem 1.2 above.

To put Theorem 1.2 into context, recall that in general energy bounds are not enough to provide bounds on the topology of holomorphic curves. Indeed, double branched covers of $\mathbb{C} P^{1}$ exist for all genera, and by choosing the branch points to lie on the equator and cutting the domain along preimages of suitable segments connecting adjacent branch points, one obtains existence of holomorphic curves of genus zero and arbitrarily many boundary components, but of fixed energy.

Often, one can use index arguments to show that such phenomena disappear after suitable perturbation. Indeed, for the Fredholm theory of holomorphic curves $f:(S, \partial S) \rightarrow(X, L)$ as above, it is convenient to puncture the source $S$ at points in $\partial S$ that map to the clean intersection and call such punctures Lagrangian intersection punctures. It turns out that to each such puncture one can associate a winding number $w \in \frac{1}{2} \mathbb{N}$, and that the contribution of a Lagrangian intersection puncture in a clean intersection 
of codimension $d$ to the Fredholm index is $1-w d$ (see the appendix for more details). Consequently, this contribution is negative provided $d \geq 3$, and equal to 0 when $d=2$ and $w=\frac{1}{2}$. It follows that for clean intersections of codimension at least three one can control the number of switches using transversality arguments. However, for codimension two - which is the most interesting case from the point of view of smooth embedding theory [5] no such argument is available. Still, the result of this paper provides a bound on the number of switches which is independent of codimension.

Similar remarks apply to the number of boundary circles $r$ and the genus $g$ of $S$ : If $\operatorname{dim}(L)=n$ satisfies $n>3$ then the number of boundary circles and the genus can be bounded using transversality arguments; this again follows from the dimension formula for the corresponding moduli spaces (see the appendix). However, if $n=3$ the dimension is independent of $g$ and $r$ and no such argument is available. Indeed, the contribution to the Gromov-Witten invariant of a Calabi-Yau three-fold of multiple covers of degree $d$ and genus $g$ of a fixed rational curve has been computed in [10]; it is nontrivial for any fixed $d \geq 2$ and arbitrarily high genus $g$, so there is no bound of the genus in terms of the degree. It would be interesting to have similar formulae for multiple covers of genus zero and many boundary components of a fixed (punctured) disc.

Our method of proof uses the integrability of $J$ near $K$ in an essential way. It would be interesting to understand to what extend the conclusion of Theorem 1.1 remains true for more general almost complex structures.

This work is organized as follows. In Sections 2 and 3 below, we collect some mostly standard background material for the convenience of the reader. The heart of the paper are Sections 4 and 5, which contain the proof of Theorems 1.1 and 1.2, respectively. In the appendix, we discuss the index formula in the setting we consider. This is not used in the proofs of the main results, but rather included to support the discussion of context given above.

After the completion of this manuscript, we were kindly informed by the authors of [13] about similar results obtained therein, see Remark 4.2 for the exact relations to our Theorem 1.1.

\section{Local theory}

Let $(S, j)$ be a connected Riemmann surface with boundary $\partial S$, possibly noncompact. We will consider functions $f: S \rightarrow \mathbb{C}$ satisfying the following conditions:

(F1) $f$ is continuous on $S$;

(F2) $f$ is holomorphic on $\operatorname{int} S:=S \backslash \partial S$;

(F3) $f$ maps $\partial S$ to $\mathbb{R} \cup \mathrm{i} \mathbb{R}$.

We start with some elementary observations. 
Lemma 2.1. A function $f$ satisfying (F1-3) is holomorphic on int $S \cup(\partial S \backslash$ $\left.f^{-1}(0)\right)$.

Proof. The function $g:=f^{2}: S \rightarrow \mathbb{C}$ is continuous on $S$, holomorphic on int $S$ and maps $\partial S$ to $\mathbb{R}$, so by the Schwarz reflection principle it is holomorphic on all of $S$. Since the square root has holomorphic branches outside zero, the result for $f$ follows.

Lemma 2.2. If $f: S \rightarrow \mathbb{C}$ satisfying (F1-3) is not identically zero, then it has only finitely many zeros in any compact subdomain $S^{\prime} \subset S$.

Proof. If not, then $g=f^{2}$ is a holomorphic function for which $g^{-1}(0)$ has a limit point, forcing it to vanish identically.

For $f: S \rightarrow \mathbb{C}$ satisfying (F1-3) and not identically zero, let $\gamma$ be a path in $S$ which does not meet any zero of $f$. Define the winding number of $f$ along $\gamma$ by

$$
w(f, \gamma):=\frac{1}{2 \pi} \int_{\gamma} f^{*} d \theta
$$

where $d \theta$ denotes the angular form on $\mathbb{C} \backslash\{0\}$.

Lemma 2.3. Suppose $f: S \rightarrow \mathbb{C}$ satisfies (F1-3) and is not identically zero. Let $S^{\prime} \subset S$ be a compact subset with piecewise smooth boundary $\partial S^{\prime}=$ $\left(S^{\prime} \cap \partial S\right) \cup \Gamma$, where $\Gamma$ is a union of disjoint arcs in $S$

not meeting any zero of $f$. Then

$$
w(f, \Gamma) \geq \#\left(f^{-1}(0) \cap \operatorname{int} S^{\prime}\right)+\frac{1}{4} \#\left(f^{-1}(0) \cap S^{\prime} \cap \partial S\right) .
$$

Proof. Around each zero $p \in \operatorname{int} S^{\prime}$ pick a small disc $D_{p} \subset \operatorname{int} S^{\prime}$ containing no other zero. Then $w\left(f, \partial D_{p}\right)=k \in \mathbb{N}$, where $(z-p)^{k}$ is the first nonvanishing term in the power series expansion of $f$ at $p$. Around each zero $q \in S^{\prime} \cap \partial S$ pick a small half-disc $D_{q}^{+} \subset S^{\prime} \backslash\left(\partial S^{\prime} \cap \operatorname{int} S\right)$ containing no other zero and set $\partial^{+} D_{q}^{+}:=\partial D_{q} \backslash \partial S$. Then $w\left(f, \partial^{+} D_{q}^{+}\right)=k / 4 \in \mathbb{N} / 4$, where $(z-q)^{k}$ is the first nonvanishing term in the power series expansion of the holomorphic function $g=f^{2}$ at $q$. Now let $S^{\prime \prime}$ be the region obtained by removing from $S^{\prime}$ all discs resp. half-discs around zeros of $f$. Since $d \theta$ is closed and the angle $f^{*} \theta$ is constant along parts of $\partial S$ containing no zeros, Stokes' theorem yields

$$
0=w\left(f, \partial S^{\prime \prime}\right)=w(f, \Gamma)-\sum_{p} w\left(f, \partial D_{p}\right)-\sum_{q} w\left(f, \partial^{+} D_{q}^{+}\right),
$$

from which the lemma follows.

Lemma 2.4. Let $f_{n}: S \rightarrow \mathbb{C}$ be a sequence of functions satisfying (F1-3), and assume that there is a constant $C>0$ such that for all $n \geq 1$ and all $z \in S$ we have

$$
\left|f_{n}(z)\right| \leq C .
$$


Then there exists a subsequence $f_{n^{\prime}}$ of the $f_{n}$, and a function $f: S \rightarrow \mathbb{C}$ satisfying (F1-3) such that

(i) $f_{n^{\prime}} \rightarrow f$ in $C_{\mathrm{loc}}^{0}$ on $S$, and

(ii) $f_{n^{\prime}} \rightarrow f$ in $C_{\mathrm{loc}}^{\infty}$ on int $S \cup\left(\partial S \backslash f^{-1}(0)\right)$.

Proof. Consider the associated sequence of holomorphic functions $g_{n}:=f_{n}^{2}$ : $S \rightarrow \mathbb{C}$. The assumptions imply that for all $z \in S$ we have

$$
\left|g_{n}(z)\right| \leq C^{2} .
$$

Hence by Montel's theorem, after passing to a subsequence, the $g_{n}$ converge in $C_{\text {loc }}^{\infty}(S)$ to a limit function $g: S \rightarrow \mathbb{C}$ which is holomorphic and maps $\partial S$ to $\mathbb{R}$. By the same argument, after passing to a further subsequence, the $f_{n}$ converge in $C_{\text {loc }}^{\infty}($ int $S)$ to a holomorphic function $f:$ int $S \rightarrow \mathbb{C}$ satisfying $f^{2}=\left.g\right|_{\text {int } S}$.

At points $z \in \partial S$ with $g(z) \neq 0$ we extend $f$ by taking the branch of $\sqrt{g}$ that agrees with $f$ at interior points near $z$, and at points $z \in \partial S$ with $g(z)=0$ we set $f(z):=0$. The resulting function $f: S \rightarrow \mathbb{C}$ satisfies (F1-3). In particular, Lemma 2.1 applies to show that $f$ is holomorphic on int $S \cup\left(\partial S \backslash f^{-1}(0)\right)$.

$C_{\text {loc }}^{0}$-convergence of the $f_{n}$ to $f$ follows from the $C_{\text {loc }}^{0}$-convergence of the $g_{n}$ to $g$ and continuity of the square root. It remains to show $C_{\mathrm{loc}}^{\infty}$-convergence $f_{n} \rightarrow f$ on compact subsets of int $S \cup\left(\partial S \backslash f^{-1}(0)\right)$. If $f \equiv 0$ this holds trivially, so suppose the $f$ does not vanish identically. Fix a compact subset $S^{\prime} \subset$ int $S \cup\left(\partial S \backslash f^{-1}(0)\right)$. By Lemma $2.2, f$ has only finitely many zeros in $S^{\prime}$. Pick a compact subset $S_{0} \subset S^{\prime} \cap$ int $S$ containing all the zeros and set $S_{1}:=S^{\prime} \backslash \operatorname{int} S_{0}$. On $S_{0}$ the $C^{\infty}$-convergence $f_{n} \rightarrow f$ was shown above, and on $S_{1}$ it follows from the $C_{\mathrm{loc}}^{\infty}$-convergence $g_{n} \rightarrow g$ and smoothness of the square root away from zero.

The following statement is a variant of a result known as Vitali's theorem.

Lemma 2.5. Let $f_{n}: S \rightarrow \mathbb{C}$ be a sequence of functions satisfying the assumptions of Lemma 2.4, and suppose there exists a compact subset $A \subset S$ such that each $f_{n}$ has at least $n$ zeros in $A$. Then the limiting function $f$ vanishes identically.

Proof. Pick a compact subset $S^{\prime} \subset S$ with piecewise smooth boundary $\partial S^{\prime}=$ $\left(S^{\prime} \cap \partial S\right) \cup \Gamma$ such that $A \subset S^{\prime} \backslash \Gamma$. If $f$ has infinitely many zeros in $S^{\prime}$, then by Lemma 2.2 it vanishes. Otherwise, after passing to a subsequence, we may assume that $f$ as well as each $f_{n}$ has only finitely many zeros in $S^{\prime}$. After slightly shrinking $S^{\prime}$ we may assume that $\Gamma$ avoids the countably many zeros of $f$ and the $f_{n}$. Since $A \subset S^{\prime} \backslash \Gamma$ and $f_{n}$ has at least $n$ zeros in $A$, Lemma 2.3 yields $w(f, \Gamma) \geq n / 4$. On the other hand, since $\left.f_{n}\right|_{\Gamma}$ converges 
smoothly to $\left.f\right|_{\Gamma}$, we have

$$
w\left(f_{n}, \Gamma\right) \stackrel{n \rightarrow \infty}{\longrightarrow} w(f, \Gamma)<\infty,
$$

contradicting the previous estimate.

\section{Global theory}

3.1. Setup. For the global theory we consider the following setup.

(X) $(X, J)$ is an almost complex manifold with cylindrical end $\mathbb{R}_{+} \times M$ adjusted to $(\omega, \lambda)$ in the sense of $[\mathbf{3}]$.

This means that $X=\bar{X} \cup\left(\mathbb{R}_{+} \times M\right)$ with $\partial \bar{X}=M,(\omega, \lambda)$ is a stable Hamiltonian structure on $M, \omega$ extends to a symplectic form on $\bar{X}$, and $J$ is compatible with $\omega$ on $\bar{X}$ and with $(\omega, \lambda)$ on $\mathbb{R}_{+} \times M$. We allow $\bar{X}$ to be noncompact but impose the following condition.

(Y) There exists a compact subset $\bar{Y} \subset \bar{X}$ such that every $J$-holomorphic map $f: S \rightarrow X$ from a compact Riemann surface with boundary satisfying $f(\partial S) \subset Y:=\bar{Y} \cup\left(\mathbb{R}_{+} \times(\bar{Y} \cap M)\right)$ is entirely contained in $Y$.

Note that condition (Y) is trivially satisfied (taking $\bar{Y}=\bar{X}$ ) if $\bar{X}$ is compact. Our assumption on the Lagrangian is the following:

(L) $L \subset Y \subset X$ is a properly immersed Lagrangian submanifold with $L \cap\left(\mathbb{R}_{+} \times M\right)=\mathbb{R}_{+} \times \Lambda$ for a compact submanifold $\Lambda \subset M$ satisfying $\left.\lambda\right|_{\Lambda}=\left.\omega\right|_{\Lambda}=0$, and such that $L$ has clean self-intersection along a compact connected submanifold $K \subset$ int $\bar{Y}$.

Here clean self-intersection means that at each point $x \in K$ exactly two branches $L_{0}, L_{1}$ of $L$ meet and $T_{x} K=T_{x} L_{0} \cap T_{x} L_{1}$. More precisely, $L$ is the image of a Lagrangian immersion $f: \tilde{L} \rightarrow X$ with clean self-intersection along the submanifold $\tilde{K}=f^{-1}(K)$. Then $\left.f\right|_{\tilde{K}}: \tilde{K} \rightarrow K$ is a $2-1$ covering and the two branches of $L$ near $x \in K$ are the images under $f$ of neighbourhoods of the preimages $x_{0}, x_{1}$ of $x$. Note that $L$ may be two-sheeted near $K$, i.e., the union of two embedded submanifolds intersecting in $K$ (if $\tilde{K}$ is disconnected), or one-sheeted (if $\tilde{K}$ is connected). We impose the following condition on the almost complex structure near $K$.

(K) There exists a neighbourhood $\mathcal{U}$ of $K$ on which $J$ is integrable, a holomorphic embedding $\iota: K^{\mathbb{C}} \hookrightarrow X$ of a complexification of $K$, and a holomorphic projection $\tau: \mathcal{U} \rightarrow K^{\mathbb{C}}$ on a neighbourhood of $K$ such that $\tau \circ \iota=\mathbb{1}$. Moreover, near every point $x \in K$ there exist holomorphic coordinates in $\mathbb{C}^{n}=\mathbb{R}^{k} \oplus \mathbb{R}^{n-k} \oplus \mathrm{i} \mathbb{R}^{k} \oplus \mathbb{i}^{n-k}$ sending $x$ to $0, L_{0}$ to $\mathbb{R}^{n}$ and $L_{1}$ to $\mathbb{R}^{k} \oplus \mathrm{i} \mathbb{R}^{n-k}$.

In particular, this implies that $L$ is real analytic near $K$ with $J$-orthogonal self-intersection along $K$, i.e., for every $x \in K$ the intersection $T_{x} L_{0} \cap$ $J\left(T_{x} L_{1}\right)$ is $(n-k)$-dimensional. However, condition $(\mathrm{K})$ is more restrictive 
than this. Indeed, not every pair of real analytic curves in $\mathbb{C}$ intersecting orthogonally at the origin can be mapped to the coordinate axes by a local biholomorphism (e.g., if one curve is the $y$-axis, then the existence of such a biholomorphism imposes infinitely many constraints on the Taylor coefficients of the other curve as a graph over the $x$-axis).

Finally, we assume that the Reeb flow on $M$ satisfies the following nondegeneracy condition:

(R) No closed Reeb orbit meets $\Lambda$, and all closed Reeb orbits and Reeb chords are nondegenerate.

Here a Reeb chord is a Reeb orbit $\gamma:[0, T] \rightarrow M$ with $\gamma(0), \gamma(T) \in \Lambda$. If there are no closed Reeb orbits (e.g., for conormal lifts of $K \subset \mathbb{R}^{n}$ with the flat metric) these conditions can be arranged by a perturbation of $\Lambda$. In the contact case $\omega=d \lambda$ these conditions can be arranged by a perturbation of $\lambda$.

Our main case of interest is described in the following example.

Example 3.1 (cotangent bundle). Here the symplectic manifold $X=T^{*} Q$ is the cotangent bundle of a Riemannian manifold $Q$ with the Liouville one-form $\lambda=p d q$ and symplectic form $\omega=d \lambda . M=S^{*} Q$ is the unit cotangent bundle and $J$ is the almost complex structure on $T^{*} Q$ induced by the Riemannian metric, deformed outside $S^{*} Q$ to make it cylindrical as described in [6, Section 7]. $K \subset Q$ is a compact submanifold and $L=$ $Q \cup N K$, where $Q$ is the zero section and $N K$ the conormal bundle, and $\Lambda=N K \cap S^{*} Q$. Then $Q$ and $N K$ intersect cleanly along $K$. We assume that $Q=\bar{Q} \cup\left(\mathbb{R}_{+} \times \partial \bar{Q}\right)$ with compact $\bar{Q}$ and $K \subset \operatorname{int} \bar{Q}$. Condition (Y) can be arranged (with $Y=T^{*} \bar{Q}$ ) by making all level sets $\{r\} \times \partial \bar{Q}$ in the cylindrical end $\mathbb{R}_{+} \times \partial \bar{Q}$ totally geodesic. Their preimages in $T^{*} Q$ are then foliated by the complex submanifolds $\{(r, s)\} \times T^{*} \bar{Q} \subset T^{*}\left(\mathbb{R}_{+} \times \bar{Q}\right) \cong$ $T^{*} \mathbb{R}_{+} \times T^{*} \bar{Q}$, hence Levi-flat, so holomorphic curves cannot touch them from inside, see [6]. Condition (R) holds for a generic metric. Condition (K) can be arranged by Proposition 3.1 below, or by Remark 3.2 if $K$ has trivial normal bundle and a neighbourhood which admits a flat metric (e.g., for a one-knot in $\mathbb{R}^{3}$ ).

3.2. Structure near $\boldsymbol{K}$. In this subsection we show that condition (K) can always be arranged by a deformation of the compatible almost complex structure near $K$, provided that $L$ is two-sheeted near $K$.

Proposition 3.1. Let $L_{0}, L_{1}$ be Lagrangian submanifolds of a symplectic $2 n$-manifold $(X, \omega)$ intersecting cleanly along a closed submanifold $K$ of dimension $k$. Then there exists an $\omega$-compatible integrable complex structure $J$ on a neighbourhood $\mathcal{U}$ of $K$ such that condition (K) holds.

The proof of this proposition is based on three lemmata. The first one provides a symplectic normal form for $L_{0}, L_{1}$ near $K$. 
Lemma 3.1. Let $L_{0}, L_{1}$ be Lagrangian submanifolds of a symplectic $2 n$ manifold $(X, \omega)$ intersecting cleanly along a closed submanifold $K$ of dimension $k$. Then there exists a symplectomorphism from a neighbourhood $\mathcal{U}$ of $K$ onto a neighbourhood of $K$ in $\left(T^{*} L_{0}, \omega_{\mathrm{st}}\right)$ mapping $L_{0}$ to the zero section and $L_{1}$ to the conormal bundle $N K$.

Proof. Consider the cotangent bundle $\pi: T^{*} L_{0} \rightarrow L_{0}$ with its standard symplectic form $\omega_{\text {st }}$. A standard application of Moser's lemma gives a symplectomorphism from a neighbourhood $(\mathcal{U}, \omega)$ of $K$ onto a neighbourhood of $K$ in $\left(T^{*} L_{0}, \omega_{\text {st }}\right)$ mapping $L_{1}$ to the conormal bundle $N K$ and such that the image $L_{0}^{\prime}$ of $L_{0}$ under this symplectomorphism is tangent to the zero section $L_{0}$ along $K$. Thus after shrinking the neighbourhood we may assume that $L_{0}^{\prime}$ is the graph of a closed one-form $\lambda$. Since $\lambda$ vanishes along $K$ it equals $d h$ for a function $h$ whose differential vanishes along $K$. The Hamiltonian flow of $h \circ \pi: T^{*} L_{0} \rightarrow \mathbb{R}$ is given by $\phi_{t}(q, p)=\left(q, p+t d_{q} h\right)$. So the time-(-1)-map $\phi_{-1}$ preserves $N K$ and maps $L_{0}^{\prime}$ to the zero section.

Next, we construct a holomorphic model for $L_{0}, L_{1}$ near $K$ for which condition (K) holds. Consider a complex vector bundle $E \rightarrow M$. A holomorphic structure on $E$ is given by the structure of complex manifolds on $E$ and $M$ together with holomorphic local trivializations. By a Kähler structure on a holomorphic vector bundle $E$ we mean a fibrewise linear Kähler form $\omega_{E}$ on E.

Lemma 3.2. Let $F \rightarrow K$ be a real vector bundle over a compact manifold $K$ and $E \rightarrow T K$ the pullback of the complexified bundle $F \otimes \mathbb{C} \rightarrow K$ to the tangent bundle $T K$. Then there exists a Kähler vector bundle structure on $E$ for which the total spaces of the subbundles $F \rightarrow K$ and $\mathrm{i} F \rightarrow K$ are real analytic, totally real and Lagrangian.

Proof. We first describe the real Kähler structures on the tautological bundles over Grassmannians. For positive integers $m<N$ consider the action of $G L(m, \mathbb{C})$ on $\mathbb{C}^{m \times N}$ by left multiplication. We think of $\mathbb{C}^{m \times N}$ as $m$-tuples of (row) vectors in $\mathbb{C}^{N}$ and denote by $\left(\mathbb{C}^{m \times N}\right)^{*}$ the subset of linearly independent tuples. The maximal compact subgroup $U(m) \subset G L(m, \mathbb{C})$ acts on $\mathbb{C}^{m \times N}$ in a Hamiltonian way (for the standard symplectic structure on $\mathbb{C}^{m \times N}$ ) with moment map

$$
\mu: \mathbb{C}^{m \times N} \rightarrow u(m), \quad M \mapsto \frac{\mathrm{i}}{2} X X^{*} .
$$

The quotient

$$
G_{\mathbb{C}}:=G_{\mathbb{C}}(m, N)=\left(\mathbb{C}^{m \times N}\right)^{*} / G L(m, \mathbb{C})=\mu^{-1}(\mathrm{i} / 2) / U(m)
$$

is the Grassmannian of $m$-dimensional complex subspaces of $\mathbb{C}^{N}$; it inherits the Kähler structure from $\mathbb{C}^{m \times N}$. 
Next consider the set

$$
V:=\left\{(X, v) \in\left(\mathbb{C}^{m \times N}\right)^{*} \times \mathbb{C}^{N} \mid v \in \operatorname{span}(X)\right\},
$$

where $\operatorname{span}(X) \subset \mathbb{C}^{N}$ denotes the complex subspace spanned by the $m$ frame $X=\left(X_{1}, \ldots, X_{m}\right)$. Since the condition $v \in \operatorname{span}(X)$ can be expressed by complex equations - the vanishing of all $(m+1)$-dimensional minors of the matrix $\left(X_{1}, \ldots, X_{m}, v\right)-V$ is a complex submanifold of $\mathbb{C}^{m \times N} \times \mathbb{C}^{N}$. The quotient

$$
\gamma_{\mathbb{C}}:=V / G L(m, \mathbb{C}) \rightarrow G_{\mathbb{C}},
$$

where $G L(m, \mathbb{C})$ acts trivially on $v \in \mathbb{C}^{N}$, is the tautological rank $m$ vector bundle over the Grassmannian $G_{\mathbb{C}}$. By construction, it inherits from $\mathbb{C}^{m \times N} \times$ $\mathbb{C}^{N}$ the structure of a Kähler vector bundle.

Complex conjugation $\sigma(X, v):=(\bar{X}, \bar{v})$ defines an anti-holomorphic (i.e., $\sigma \circ i=-i \circ \sigma$ ) and anti-symplectic (i.e., $\sigma^{*} \omega_{\text {st }}=-\omega_{\text {st }}$ ) involution of $\mathbb{C}^{m \times N}$. Since $\sigma(U X, v)=\bar{U} \sigma(X, v)$ it descends to an anti-holomorphic and anti-symplectic involution on $\gamma_{\mathbb{C}}$. Its fixed point set, the total space of the tautological bundle

$$
\gamma_{\mathbb{R}} \rightarrow G_{\mathbb{R}}:=G_{\mathbb{R}}(m, N)
$$

over the Grassmannian of real $m$-planes in $\mathbb{R}^{N}$ is therefore real analytic, totally real and Lagrangian. The map $I(X, v):=(X, \mathrm{i} v)$ on $\mathbb{C}^{m \times N} \times \mathbb{C}^{N}$ is holomorphic and symplectic. Since it commutes with the action of $G L(m, \mathbb{C})$ and satisfies $I \circ \sigma=-\sigma \circ I$, it descends to a holomorphic and symplectic map on $\gamma_{\mathbb{C}}$ which anti-commutes with $\sigma$. Thus the total space of the bundle

$$
\mathrm{i} \gamma_{\mathbb{R}}:=I\left(\gamma_{\mathbb{R}}\right) \rightarrow G_{\mathbb{R}}
$$

(whose fibre over a real subspace $W \subset \mathbb{R}^{N}$ is the subspace $\mathrm{i} W \subset \mathbb{C}^{N}$ ) is also real analytic, totally real and Lagrangian.

Now let $F \rightarrow K$ be a real vector bundle of rank $m$ over a compact manifold $K$. Then for sufficiently large $N$ there exists a continuous map $\phi: K \rightarrow G_{\mathbb{R}}$ such that $F \cong \phi^{*} \gamma_{\mathbb{R}}$. We equip $K$ with a real analytic structure. Complexification yields a complex structure on the total space of the tangent bundle $T K$ such that the zero section is real analytic (after replacing $T K$ by a neighbourhood of the zero section and identifying this again with $T K$ ). We approximate $\phi$ by a real analytic embedding into $G_{\mathbb{R}}$ (which is possible for $N$ large) and complexify this to a holomorphic embedding $\phi_{\mathbb{C}}: T K \hookrightarrow G_{\mathbb{C}}$. By construction, $\phi$ is covered by an injective bundle map $\Phi: F \rightarrow \gamma_{\mathbb{R}}$. We complexify it to an injective bundle map $F \otimes \mathbb{C} \rightarrow \gamma_{\mathbb{C}}$ mapping i $F$ to i $\gamma_{\mathbb{R}}$ and extend it to an injective bundle map $\Phi_{\mathbb{C}}: E \rightarrow \gamma_{\mathbb{C}}$ covering $\phi_{\mathbb{C}}$. Now the Kähler bundle structure on $\left.\gamma_{\mathbb{C}}\right|_{\phi_{\mathbb{C}}(T K)}$ pulls back under $\Phi_{\mathbb{C}}$ to a Kähler bundle structure on $E \rightarrow T K$ with the desired properties. 
Lemma 3.3. Let $E \rightarrow T K$ be as in Lemma 3.2 with $\operatorname{dim} K=k$ and rank $E=n-k$. Then hypothesis $(\mathrm{K})$ is satisfied for $X=E, L_{0}=F$ and $L_{1}=\mathrm{i} F$.

Proof. For the holomorphic vector bundle $\tau: E \rightarrow T K$, the projection $\tau$ and the inclusion $\iota: T K \hookrightarrow E$ of the zero section are holomorphic. Next consider $x \in K$. Pick a neighbourhood $V$ of $x$ in $K$ and a real analytic trivialization $\phi:\left.F\right|_{V} \hookrightarrow \mathbb{R}^{k} \times \mathbb{R}^{n-k}$ mapping $x$ to 0 . Complexify it to a holomorphic embedding $\phi^{\mathbb{C}}: \mathcal{N} \hookrightarrow \mathbb{C}^{k} \times \mathbb{C}^{n-k}$ of a neighbourhood $\mathcal{N}$ of $\left.F\right|_{V}$ in $E$. By uniqueness of analytic continuation, the restriction of $\phi^{\mathbb{C}}$ to each $E_{x} \cap \mathcal{N}$ with $x \in V$ is complex linear and we can extend it linearly to the whole fibre $E_{x}$. Thus we may assume that $\mathcal{N}$ contains $\left.E\right|_{V}$. By construction, $\phi^{\mathbb{C}}$ maps $\left.F\right|_{V}$ to $\mathbb{R}^{k} \times \mathbb{R}^{n-k}$, and by complex linearity in the fibres it maps $\left.\mathrm{i} F\right|_{V}$ to $\mathbb{R}^{k} \times \mathrm{i} \mathbb{R}^{n-k}$.

Proof of Proposition 3.1. Let $(X, \omega), L_{0}, L_{1}$ and $K$ be as in the proposition. Let $F \rightarrow K$ be the normal bundle of $K$ in $L_{0}$ and denote by $E \rightarrow T K$ the pullback bundle of $F \otimes \mathbb{C} \rightarrow K$ under the projection $T K \rightarrow K$ as in Lemma 3.2. Then a neighbourhood of $K$ in $X$ is diffeomorphic to a neighbourhood of $K$ in $E$ such that $L_{0}$ corresponds to $F$ and $L_{1}$ to i $F$. Lemma 3.2 provides a Kähler vector bundle structure on $E$, with Kähler form $\omega_{E}$, for which $F$ and i $F$ are Lagrangian. By Lemma 3.1, the quadruples $\left(X, \omega, L_{0}, L_{1}\right)$ and $\left(E, \omega_{E}, F, \mathrm{i} F\right)$ are both isomorphic near $K$ to the same standard model. Hence there exists a symplectomorphism from a neighbourhood $\mathcal{U}$ of $K$ in $(X, \omega)$ to a neighbourhood of $K$ in $\left(E, \omega_{E}\right)$ mapping $L_{0}$ to $F$ and $L_{1}$ to i $F$. The holomorphic structure on $E$ pulls back to an $\omega$-compatible integrable complex structure $J$ on $\mathcal{U}$, which satisfies condition (K) by Lemma 3.3 .

Remark 3.1. Proposition 3.1 should also hold if $L$ is one-sheeted near $K$, but the proof will be more involved in that case.

Remark 3.2. Consider a submanifold $K \subset Q$ and the immersed Lagrangian $L=Q \cup N K \subset T^{*} Q$ as in Example 3.1. Suppose that $K$ has trivial normal bundle and a neighbourhood which admits a flat metric (e.g., for a oneknot in $\mathbb{R}^{3}$ ). Choose $J$ to agree with the (integrable!) complex structure on a neighbourhood of $K$ in $T^{*} Q$ induced by this metric. After a small perturbation, we may assume that $K \subset Q$ is real analytic. We claim that $K$ then satisfies condition $(\mathrm{K})$.

To see this, pick linearly independent real analytic normal vector fields $v_{1}, \ldots, v_{n-k}$ along $K$. They induce a real analytic embedding

$$
I: K \times B \hookrightarrow Q, \quad\left(q, s_{1}, \ldots, s_{n-k}\right) \mapsto q+s_{1} v_{1}(q)+\ldots+s_{n-k} v_{n-k}(q) .
$$

Here $B$ is a small ball around the origin in $\mathbb{R}^{n-k}$ and the right-hand side is to be understood in flat coordinates near $q$. Intrinsically, it can be described as 
moving from $q$ for time $s_{1}$ along the geodesic in direction $v_{1}(q)$, then for time $s_{2}$ along the geodesic in direction $v_{2}(q)$ (parallel transported to $q+s_{1} v_{1}(q)$ ), etc. This embedding complexifies to a holomorphic embedding

$$
\begin{aligned}
I^{\mathbb{C}}: K^{\mathbb{C}} \times B^{\mathbb{C}} & \hookrightarrow T^{*} Q, \quad\left(x, z_{1}, \ldots, z_{n-k}\right) \\
& \mapsto \iota(x)+z_{1} v_{1}^{\mathbb{C}}(x)+\ldots+z_{n-k} v_{n-k}^{\mathbb{C}}(x),
\end{aligned}
$$

where $\iota: K^{\mathbb{C}} \hookrightarrow T^{*} Q$ is the complexification of the inclusion $K \hookrightarrow Q$ and $v_{j}^{\mathbb{C}}$ is the complexification of $v_{j}$ viewed as a map $K \rightarrow \mathbb{R}^{n}$ in flat local coordinates. This shows the existence of the holomorphic embedding $\iota$ and projection $\tau$ in $(\mathrm{K})$. For the last property, note that the restriction of $I^{\mathbb{C}}$ to $K \times \mathrm{i} B$ is given by

$$
\left(q, \mathrm{i} t_{1}, \ldots, \mathrm{i} t_{n-k}\right) \mapsto q+\mathrm{i} t_{1} v_{1}(q)+\ldots+\mathrm{i} t_{n-k} v_{n-k}(q) .
$$

Thus $I^{\mathbb{C}}$ maps $K \times \mathrm{i} B$ to the conormal bundle $L_{1}=N K$ and $K \times B$ to the zero section $L_{0}=Q$, so the desired local coordinates in $(\mathrm{K})$ are induced by $\left(I^{\mathbb{C}}\right)^{-1}$ and flat coordinates on $Q$.

For the remainder of this section, we consider $(X, L, \omega, J)$ satisfying conditions $(\mathrm{X}),(\mathrm{Y}),(\mathrm{L}),(\mathrm{K})$ and $(\mathrm{R})$ above.

3.3. Area and energy. Recall from [3] that the (Hofer) energy of a holomorphic curve $f$ is defined as a sum of two terms,

$$
E(f):=E_{\omega}(f)+E_{\lambda}(f) .
$$

When $f=\left(f_{\mathbb{R}}, f_{M}\right):(S, \partial S, j) \rightarrow(\mathbb{R} \times M, \mathbb{R} \times \Lambda, J)$, we set

$$
E_{\omega}(f):=\int_{S} f_{M}^{*} \omega, \quad E_{\lambda}(f):=\sup _{\varphi \in \mathcal{C}} \int_{S}\left(\varphi \circ f_{\mathbb{R}}\right) d f_{\mathbb{R}} \wedge f_{M}^{*} \lambda
$$

where the supremum is taken over the $\operatorname{set} \mathcal{C}$ of nonnegative functions $\varphi$ : $\mathbb{R} \rightarrow \mathbb{R}$ with

$$
\int_{\mathbb{R}} \varphi(s) d s=1 .
$$

Similarly, for a holomorphic curve $f:(S, \partial S, j) \rightarrow(X, L, J)$ we define its $\omega$-energy (or area)

$$
E_{\omega}(f):=\int_{f^{-1}(\bar{X})} f^{*} \omega+\int_{f^{-1}\left(\mathbb{R}_{+} \times M\right)} f_{M}^{*} \omega
$$

and its $\lambda$-energy

$$
E_{\lambda}(f):=\sup _{\varphi \in \mathcal{C}^{+}} \int_{f^{-1}\left(\mathbb{R}_{+} \times M\right)}\left(\varphi \circ f_{\mathbb{R}}\right) d f_{\mathbb{R}} \wedge f_{M}^{*} \lambda,
$$

where the supremum is taken over the set $\mathcal{C}^{+}$of all nonnegative functions $\varphi: \mathbb{R}_{+} \rightarrow \mathbb{R}$ with

$$
\int_{\mathbb{R}^{+}} \varphi(s) d s=1
$$


Since the almost complex structure is compatible with $\omega$ and since $J$ pairs the symplectization- and the Reeb direction in the ends of $X$, it follows that $E_{\omega}(f) \geq 0$ and $E_{\lambda}(f) \geq 0$ for any holomorphic $f$. Moreover, $E_{\omega}(f)=0$ implies that either $f$ is constant, or the image of $f$ is contained in some cylinder over a closed Reeb orbit or in some strip over a Reeb chord.

\subsection{Monotonicity and removal of singularities.}

Lemma 3.4 (Monotonicity Lemma). There exist constants $\varepsilon_{M}, C_{M}>0$ depending only on $(X, L, \omega, J)$ with the following property: For any nonconstant $J$-holomorphic map $f:(S, \partial S) \rightarrow(X, L)$ from a (possibly noncompact) Riemann surface with boundary, passing through a point $x \in \bar{Y}$ and such that $f^{-1}(B(x, r))$ is compact for some $r<\varepsilon_{M}$, we have

$$
E_{\omega}(f) \geq C_{M} r^{2}
$$

Proof. The proof in Proposition 4.7.2 in [18] carries over to the present setting as follows. Since the metric is smooth and $K$ is compact, there exist constants $C_{0}, C_{1}, C_{2}$, and $\varepsilon_{M}>0$ with the following properties. For $0<$ $r<\varepsilon_{M}$ and any $x \in \bar{Y}: B(x, r) \cap L$ is contained in a contractible subset of $B\left(x, C_{2} r\right) \cap L$, for every pair of points $y, z \in B(x, r) \cap L$ there is a curve in $B\left(x, C_{2} r\right) \cap L$ of length at most $C_{1} d(y, z)$ connecting them, and every closed curve $\gamma$ in $B\left(x, C_{2} r\right)$ bounds a disc in $B\left(x, C_{2} r\right)$ of area at most $C_{0} \ell^{2}(\gamma)$, where $\ell(\gamma)$ denotes the length of $\gamma$.

Assume that $x \in f(S)$. For $r<\varepsilon_{M}$, let $S_{r}=f(S) \cap B(x, r)$ and decompose $\partial S_{r}=\alpha_{r} \cup \beta_{r}$, where $\alpha_{r}=\partial B(x, r) \cap f(S)$ and $\beta_{r}=f(\partial S) \cap B(x, r)$. If $r$ is chosen generically then $\alpha_{r}$ and $\beta_{r}$ are collections of smooth curves, with $\beta_{r}$ possibly empty. For each non-closed component $\alpha_{r}^{\prime}$ of $\alpha_{r}$ we choose a curve $\gamma_{r}^{\prime}$ in $L \cap B\left(x, C_{2} r\right)$ which connects its endpoints and is of length at most $C_{1} l\left(\alpha_{r}^{\prime}\right)$, where $l\left(\alpha_{r}^{\prime}\right)$ is the length of $\alpha_{r}^{\prime}$. Then $\alpha_{r}^{\prime} \cup \gamma_{r}^{\prime}$ is a closed curve in $B\left(x, C_{2} r\right)$ of length at most $\left(1+C_{1}\right) l\left(\alpha_{r}^{\prime}\right)$. Let $\gamma_{r}$ denote the union of all curves $\gamma_{r}^{\prime}$. Then by assumption $\alpha_{r} \cup \gamma_{r}$ bounds a collection $D$ of discs in $B(x, r)$ of total area at most $C l^{2}\left(\alpha_{r}\right)$. Similarly, $\beta_{r} \cup \gamma_{r}$, if nonempty, is a cycle in the contractible set $B\left(x, C_{2} r\right) \cap L$, and so it bounds a surface $N$ in $L \cap B\left(x, C_{2} r\right)$. By Stokes' theorem

$$
\int_{S_{r} \cup D \cup N} \omega=0 .
$$

Clearly $\int_{N} \omega=0$. Moreover, since $\omega$ is a calibration, $\left|\int_{D} \omega\right|$ is bounded by the area of $D$, and we conclude that

$$
\int_{S_{r}} \omega \leq C \ell^{2}\left(\alpha_{r}\right)
$$

for some constant $C$. Consider the distance function $\rho$ from $x$. Since the norm of the gradient of $\rho$ in the ambient manifold is 1 we conclude that $|\nabla \rho| \leq 1$ on $S_{r}$. So if we let $a(\rho)$ denote the area of $S_{\rho}$ then, by Sard's 
theorem and the coarea formula, we have $a^{\prime}(\rho) \geq \ell\left(\alpha_{\rho}\right)$ for almost every $\rho \leq r$. Consequently

$$
\frac{d \sqrt{a}}{d \rho}=\frac{a^{\prime}(\rho)}{2 \sqrt{a(\rho)}} \geq \frac{1}{2 \sqrt{C^{\prime}}},
$$

for some constant $C^{\prime}$. Integrating we find $a(r) \geq C_{M} r^{2}$.

Remark 3.3. Alternatively, Lemma 3.4 can be proved by using Proposition 4.7.2 in [18] outside $K$ and condition $(\mathrm{K})$ near $K$. This reduces the lemma to the case of a holomorphic map $f=\left(f_{1}, \ldots, f_{n}\right):(S, \partial S) \rightarrow$ $\left(\mathbb{C}^{n}, \mathbb{R}^{n} \cup \mathbb{R}^{k} \times \mathrm{i} \mathbb{R}^{n-k}\right)$ passing through the origin, which can be proved by considering the componentwise square $g:=\left(f_{1}^{2}, \ldots, f_{n}^{2}\right):(S, \partial S) \rightarrow\left(\mathbb{C}^{n}, \mathbb{R}^{n}\right)$ with smooth Lagrangian boundary condition.

Let $D:=\{z \in \mathbb{C}|| z \mid<1\}$ and $D^{+}:=\{z \in D \mid \mathfrak{I m}(z) \geq 0\}$.

Lemma 3.5 (Removal of singularities). (a) Let $f: D \backslash\{0\} \rightarrow X$ be continuous and $J$-holomorphic in the interior with finite energy $E(f)<\infty$. If $f$ is bounded, then it extends to a continuous map $D \rightarrow X$.

(b) Let $f:\left(D^{+} \backslash\{0\}, D^{+} \cap \mathbb{R} \backslash\{0\}\right) \rightarrow(X, L)$ be continuous and $J$ holomorphic in the interior with finite energy $E(f)<\infty$. If $f$ is bounded, then it extends to a continuous map $D^{+} \rightarrow X$.

Proof. Both cases follow from the argument given right after Theorem 4.1.2 in [15], using the Monotonicity Lemma 3.4 above.

3.5. Asymptotics. We have the following descriptions of the asymptotic behaviour of a holomorphic curve $f:(S, \partial S) \rightarrow(X, L)$ where $(X, L, \omega, J)$ satisfies conditions $(\mathrm{X}),(\mathrm{Y}),(\mathrm{L}),(\mathrm{K})$ and $(\mathrm{R})$ above near a nonremovable puncture (cf. [3, Proposition 5.6]).

Proposition 3.1. (a) Let $f: \mathbb{R}_{+} \times S^{1} \rightarrow(Y, J)$ be a holomorphic curve with $E(f)<\infty$ and suppose the image of $f$ is unbounded. Then $f(s, t) \in \mathbb{R}_{+} \times M$ for all sufficiently large $s$, and there exists $T>0$ and a periodic orbit $\gamma$ of the Reeb vector field of period $T$ such that

$$
\lim _{s \rightarrow \infty} \pi_{M} \circ f(s, t)=\gamma(T t), \quad \lim _{s \rightarrow \infty} \frac{\pi_{\mathbb{R}} \circ f(s, t)}{s}=T
$$

in $C^{\infty}\left(S^{1}\right)$.

(b) Let $f:\left(\mathbb{R}_{+} \times[0,1], \mathbb{R}_{+} \times\{0,1\}\right) \rightarrow(Y, L, J)$ be a holomorphic curve with $E(f)<\infty$ and suppose the image of $f$ is unbounded. Then $f(s, t) \in$ $\mathbb{R}_{+} \times M$ for all sufficiently large $s$, and there exists $T>0$ and a Reeb chord $\gamma$ of $\Lambda \subset M$ of length $T$ such that

$$
\lim _{s \rightarrow \infty} \pi_{M} \circ f(s, t)=\gamma(T t), \quad \lim _{s \rightarrow \infty} \frac{\pi_{\mathbb{R}} \circ f(s, t)}{s}=T
$$

in $C^{\infty}([0,1])$. 
Proof. Case (a) is proved in [11]. Case (b) is proved in $[\mathbf{1}]$ in case $\operatorname{dim}(Y)=3$ and the proof there carries over to the higher dimensional situation with only minor changes.

\subsection{Quantization of energy.}

Lemma 3.6. There exists a constant $\hbar>0$, depending only on $(X, L, \omega, J)$, such that for every proper $J$-holomorphic map $f:(S, \partial S) \rightarrow(X, L)$

$$
E_{\omega}(f) \geq \hbar \text {. }
$$

Proof. The proof of Lemma 4.2 in [7] for symplectizations directly carries over to the relative case, giving the result for curves whose image is contained in the end $\mathbb{R}_{+} \times M$. For curves whose image meets $\bar{X}$, the lower energy bound is guaranteed by the Monotonicity Lemma 3.4.

Lemma 3.7. For every $E>0$ there exists a constant $\hbar(E)>0$, depending only on $(X, L, \omega, J)$ and $E$, such that the area of every proper $J$-holomorphic cylinder or strip $f:(S, \partial S) \rightarrow(X, L)$ with $E(f) \leq E$ and $E_{\omega}(f)>0$ satisfies

$$
E_{\omega}(f) \geq \hbar(E) \text {. }
$$

Proof. The proof of Lemma 10.9 in [3] resp. Lemma 4.6 in [7] carries over to the relative case.

3.7. Holomorphic cylinders and strips of small area. Finally, we need the following generalization of a result of Hofer, Wysocki and Zehnder.

Proposition 3.2. Given $E_{0}, \varepsilon>0$ there are constants $\sigma, c>0$ with the following properties:

(a) For every $R>c$ and every holomorphic cylinder $f:[-R, R] \times S^{1} \rightarrow$ $\mathbb{R} \times M$ satisfying $E_{\omega}(f) \leq \sigma$ and $E(f) \leq E_{0}$ there exists either a periodic Reeb orbit $\gamma$ of period $T>0$ such that $\pi_{M} \circ f(s, t) \in B_{\varepsilon}(\gamma(T t))$ or some point $p \in M$ such that $\pi_{M} \circ f(s, t) \in B_{\varepsilon}(p)$ for all $s \in[-R+c, R-c]$ and all $t \in S^{1}$.

(b) For every $R>c$ and every holomorphic strip $f:([-R, R] \times[0,1]$, $[-R, R] \times\{0,1\} \rightarrow(\mathbb{R} \times M, \mathbb{R} \times \Lambda)$ satisfying the inequalities $E_{\omega}(f) \leq \sigma$ and $E(f) \leq E_{0}$ there exists either a Reeb chord $\gamma$ of length $T>0$ such that $\pi_{M} \circ f(s, t) \in B_{\varepsilon}(\gamma(T t))$ or some point $p \in \Lambda$ such that $\pi_{M} \circ f(s, t) \in B_{\varepsilon}(p)$ for all $s \in[-R+c, R-c]$ and all $t \in[0,1]$.

Proof. The proof of (a) in [12] (for the contact case) and [3] (for general stable Hamiltonian structures) carries over to case (b).

We will also use the following version of Lemma 5.14. of [3], whose proof carries over to the relative case using the Monotonicity Lemma 3.4.

Lemma 3.8. Let $u_{n}:([-n, n] \times[0,1],[-n, n] \times\{1,0\}) \rightarrow(X, L)$ be a sequence of $J$-holomorphic strips with 
(i) $\lim _{n \rightarrow \infty} E_{\omega}\left(u_{n}\right)=0$, and

(ii) $\left.\lim _{n \rightarrow \infty} u_{n}\right|_{\{ \pm n\} \times[0,1]}=p_{ \pm} \in L$ in $C^{\infty}([0,1], X)$.

Then $\lim _{n \rightarrow \infty} \operatorname{diam} u_{n}([-n, n] \times[0,1])=0$, and in particular $p_{+}=p_{-}$.

\section{Compactness}

In this section, we apply the above local theory to establish a compactness result for holomorphic curves. We consider $(X, L, \omega, J)$ satisfying conditions $(\mathrm{X}),(\mathrm{Y}),(\mathrm{L}),(\mathrm{K})$ and $(\mathrm{R})$ from the previous section. Without loss of generality, we assume that the neighbourhood in condition $(\mathrm{K})$ is $\mathcal{U}=\mathcal{U}_{\varepsilon}$, where $\mathcal{U}_{r}$ denotes the open $r$-neighbourhood of $K$ in $X$ with respect to the distance induced by $(\omega, J)$.

4.1. $C_{\text {loc }}^{\infty}$-convergence. Consider a fixed connected Riemann surface $(\Sigma, j)$ of finite type, by which we mean the complement of a finite set of points (the "punctures") in a connected compact Riemann surface with boundary. We assume that $\Sigma$ is stable, meaning that its double is a stable punctured Riemann surface in the usual sense. It follows that $\Sigma$ admits a unique complete hyperbolic metric $h_{j}$ compatible with $j$ such that each component of $\partial \Sigma$ is a geodesic (either closed or infinite).

Suppose $f_{n}:(\Sigma, \partial \Sigma, j), \rightarrow(Y, L, J)$ is a sequence of continuous maps which are $J$-holomorphic on int $\Sigma \cup\left(\partial \Sigma \backslash f_{n}^{-1}(K)\right)$ and have finite energy. Recall (Proposition 3.1) that if an interior puncture is a nonremovable singularity of the map $f_{n}$, then $f_{n}$ will be asymptotic to a trivial cylinder over a closed Reeb orbit in a neighbourhood of that puncture. Similarly, near nonremovable boundary punctures $f_{n}$ is asymptotic to a trivial strip over a Reeb chord. We make the following additional assumptions on our sequence:

(S1) Each puncture is either removable for all $n \geq 1$ or nonremovable for all $n \geq 1$, and at nonremovable punctures the asymptotic Reeb chords resp. closed Reeb orbits are independent of $n$.

(S2) There exists a constant $C>1$ such that for all $z \in$ int $\Sigma$ and all $n \geq 1$ we have

$$
\begin{gathered}
\left|\nabla f_{n}(z)\right| \leq \frac{C}{\rho(z)} \quad \text { if } f_{n}(z) \notin \mathcal{U}_{\varepsilon / 4}, \\
\left|\nabla\left(\tau \circ f_{n}\right)(z)\right| \leq \frac{C}{\rho(z)} \quad \text { if } f_{n}(z) \in \mathcal{U}_{\varepsilon} .
\end{gathered}
$$

Here $\rho(z)$ denotes the injectivity radius at $z \in \Sigma$ in the hyperbolic metric, the norm is computed with respect to the hyperbolic metric on the domain and the metric determined by $\omega$ and $J$ on the target, and $\tau: \mathcal{U}_{\varepsilon} \rightarrow T^{*} K$ is the holomorphic projection appearing in condition $(\mathrm{K})$.

We denote by $\sigma_{t}: \mathbb{R} \times M \rightarrow \mathbb{R} \times M$ the map which shifts the $\mathbb{R}$-component by $t$. 
Proposition 4.1. For any sequence of maps $f_{n}:(\Sigma, \partial \Sigma, j) \rightarrow(Y, L, J)$ satisfying (S1) and (S2) there exists a subsequence, still denoted $f_{n}$, such that one of following holds:

(i) There exists a holomorphic map $f:(\Sigma, \partial \Sigma, j) \rightarrow(\mathbb{R} \times M, \mathbb{R} \times \Lambda, J)$, and constants $t_{n} \in \mathbb{R}$, such that for any compact subset $A \subset \Sigma$ there exists $N(A)$ such that for all $n \geq N(A)$ we have $f_{n}(A) \subset \mathbb{R}_{+} \times M$ and $\left.\left.\sigma_{t_{n}} \circ f_{n}\right|_{A} \rightarrow f\right|_{A}$ in $C^{\infty}$. In other words, the sequence of maps $f_{n}$ converges in the SFT sense to a curve in the symplectization.

(ii) There exists a continuous map $f:(\Sigma, \partial \Sigma, j) \rightarrow(Y, L, J)$ which is holomorphic on int $\Sigma \cup\left(\partial \Sigma \backslash f^{-1}(K)\right)$ such that $f_{n} \rightarrow f$ in $C_{\mathrm{loc}}^{0}$ on $\Sigma$, and $f_{n} \rightarrow f$ in $C_{\mathrm{loc}}^{\infty}$ on int $\Sigma \cup\left(\partial \Sigma \backslash f^{-1}(K)\right)$.

Proof. We fix an exhaustion $B_{1} \subset B_{2} \subset B_{3} \subset \cdots$ of $\Sigma$ by closed subsets $B_{j}:=\left\{z \in \Sigma: d\left(z_{0}, z\right) \leq j\right\}$, where $d\left(z_{0}, z\right)$ denotes the distance between some fixed point $z_{0}$ and $z$. Since $B_{j}$ is compact, the injectivity radius $\rho$ is bounded below on it by

$$
\rho_{j}:=\min _{B_{j}} \rho>0 .
$$

So with $C_{j}:=C / \rho_{j}$ condition (S2) yields the following gradient bounds for $z \in B_{j} \cap$ int $\Sigma$ and all $n$ :

$$
\begin{gathered}
\left|\nabla f_{n}(z)\right| \leq C_{j} \quad \text { if } f_{n}(z) \notin \mathcal{U}_{\varepsilon / 4}, \\
\left|\nabla\left(\tau \circ f_{n}\right)(z)\right| \leq C_{j} \quad \text { if } f_{n}(z) \in \mathcal{U}_{\varepsilon} .
\end{gathered}
$$

We now distinguish two cases.

Case 1. The sequence $f_{n}\left(z_{0}\right)$ is unbounded.

Then, after passing to a subsequence, we have $f_{n}\left(z_{0}\right) \in \mathbb{R}_{+} \times M$ with $\mathbb{R}$ component going to infinity. By the gradient bounds on $B_{j}$, for each fixed $j$ we have $f_{n}\left(B_{j}\right) \subset \mathbb{R}_{+} \times M$ for all sufficiently large $n$ with $\mathbb{R}$-component going uniformly to infinity. Hence we can apply the usual compactness argument with smooth Lagrangian boundary conditions $\mathbb{R} \times \Lambda$ in the symplectization $\mathbb{R} \times M$ to obtain a limiting map $f$ with image in $\mathbb{R} \times M$ as in the first case of the proposition.

Case 2. The sequence $f_{n}\left(z_{0}\right)$ remains in a compact subset $A \subset X$.

By the gradient bounds on $B_{j}$, for each fixed $j$ the images $f_{n}\left(B_{j}\right)$ remain in the compact subset

$$
A_{j}:=\left\{x \in X \mid d\left(x, A \cup \overline{\mathcal{U}}_{\varepsilon}\right) \leq C_{j} j\right\} .
$$

For each $z \in B_{j}$, we define the open ball

$$
S_{z}:=\operatorname{int} B\left(z, \frac{\varepsilon}{4 C_{j}}\right) \text {. }
$$

Now $B_{j}$ is covered by a finite collection $S_{z_{1}}, \ldots, S_{z_{r}}$ of these sets. 
For each of the points $z_{i}$, exactly one of the following two things happens:

(a) after passing to a subsequence $n_{k}, f_{n_{k}}\left(z_{i}\right) \notin \mathcal{U}_{\varepsilon / 2}$ for all $k \geq 1$, or

(b) there exists some $N\left(z_{i}\right)$ such that $f_{n}\left(z_{i}\right) \in \mathcal{U}_{\varepsilon / 2}$ for all $n \geq N\left(z_{i}\right)$.

If $z_{1}$ is of type (a), then we pass to the subsequence $f_{n_{k}}$, and if $z_{1}$ is of type (b) we pass to the subsequence $f_{n}$ with $n \geq N\left(z_{1}\right)$. Repeating this for each index $i=2, \ldots, r$, we arrive at the situation where for each $z_{i}$ either (a) or (b) holds for all $n \geq 1$.

Consider first $z_{i}$ of type (a). Then the gradient bounds imply that $f_{n}\left(S_{z_{i}}\right) \cap \mathcal{U}_{\varepsilon / 4}=\varnothing$ for all $n \geq 1$. So the maps $f_{n}: S_{z_{i}} \rightarrow X$ have smooth Lagrangian boundary conditions, and the usual compactness argument yields a subsequence which converges in $C_{\mathrm{loc}}^{\infty}$ (up to the boundary) to a holomorphic limit map.

Next we consider $z_{i}$ of type (b). We claim that $f_{n}\left(S_{z_{i}}\right) \subset \mathcal{U}_{\varepsilon}$ for all $n \geq 1$. To see this, consider $z \in S_{z_{i}}$ and a constant speed minimal geodesic $\gamma$ : $[0,1] \rightarrow \Sigma$ from $z_{i}$ to $z$, set

$$
t^{\prime}:=\sup \left\{t \in[0,1]: d\left(f_{n}(\gamma(t)), K\right) \leq \frac{\varepsilon}{2}\right\},
$$

and compute

$$
\begin{aligned}
d\left(f_{n}(z), K\right) & \leq \frac{\varepsilon}{2}+d\left(f_{n}\left(\gamma\left(t^{\prime}\right)\right), f_{n}(z)\right) \\
& \leq \frac{\varepsilon}{2}+\int_{t^{\prime}}^{1}\left|\nabla f_{n}(\gamma(t))\right||\dot{\gamma}(t)| d t \\
& \leq \frac{\varepsilon}{2}+d\left(z_{i}, z\right) \max _{t \in\left[t^{\prime}, 1\right]}\left|\nabla f_{n}(\gamma(t))\right| \\
& \leq \frac{\varepsilon}{2}+\frac{\varepsilon}{4 C_{j}} C_{j} \\
& \leq \varepsilon .
\end{aligned}
$$

This proves the claim. Now consider the holomorphic maps

$$
\tau \circ f_{n}: S_{z_{i}} \rightarrow T^{*} K
$$

where $\tau: \mathcal{U}_{\varepsilon} \rightarrow T^{*} K$ is the holomorphic projection in condition (K). These maps have smooth Lagrangian boundary conditions on $K$, are uniformly bounded, and have uniform gradient bounds by condition (4.4) above. So the usual compactness argument yields a convergent subsequence. Denote the limit map by $g: S_{z_{i}} \rightarrow T^{*} K$.

It remains to show convergence of the components transverse to $T^{*} K$. For this, note that by condition (K) each $x \in T^{*} K$ has a neighbourhood $U_{x}$ with a holomorphic embedding $\tau^{-1}\left(U_{x}\right) \hookrightarrow U_{x} \times \mathbb{C}^{n-k}$ sending the branches of $L$ to $K \times \mathbb{R}^{n-k}$ and $K \times \mathrm{i} \mathbb{R}^{n-k}$, where $k=\operatorname{dim} K$. Cover the image of $\tau$ by finitely many such neighbourhoods $U_{x_{1}}, \ldots, U_{x_{s}}$ and denote by $\nu_{\ell}$ : $U_{x} \times \mathbb{C}^{n-k} \rightarrow \mathbb{C}$ the holomorphic projection onto the $\ell$ th $\mathbb{C}$-factor, $\ell=$ $1, \ldots, n-k$. Pulling back the $U_{x_{m}}$ under $g$ yields open subsets $S_{z_{i}, m} \subset S_{z_{i}}$ and 
holomorphic functions $\nu_{\ell} \circ f_{n}: S_{z_{i}, m} \rightarrow \mathbb{C}$ mapping the boundary $S_{z_{i}, m} \cap \partial \Sigma$ to $\mathbb{R} \cup i \mathbb{R}$. Since the functions are also uniformly bounded, Lemma 2.4 yields a convergent subsequence for each $\ell=1, \ldots, n-k$ and $m=1, \ldots, s$.

Combining types (a) and (b), we conclude that $f_{n}: B_{j} \rightarrow X$ has a subsequence converging in the desired sense to a continuous limit map $f_{j}$ : $\left(B_{j}, B_{j} \cap \partial \Sigma\right) \rightarrow(X, L)$ which is holomorphic on $B_{j} \cap\left(\operatorname{int} \Sigma \cup\left(\partial \Sigma \backslash f_{j}^{-1}(K)\right)\right)$. Finally, we take a diagonal sequence with respect to the index $j$ of $B_{j}$ in our exhaustion to get a subsequence converging on all of $\Sigma$ to a limit map $f:(\Sigma, \partial \Sigma) \rightarrow(X, L)$, with convergence in $C_{\mathrm{loc}}^{0}$ on $\Sigma$ and in $C_{\mathrm{loc}}^{\infty}$ on int $\Sigma \cup$ $\left(\partial \Sigma \backslash f^{-1}(K)\right)$ as in the second case of the proposition.

Remark 4.1. Note that the same proof works if we allow the domains of $f_{n}$ to vary in a converging sequence of Riemann surfaces $\left(\Sigma_{n}, j_{n}\right)$.

4.2. Proof of the Compactness Theorem 1.1. For the remainder of this section, we assume familiarity with the proof of compactness for holomorphic curves in SFT presented in [3], and we will sketch how it can be adapted to our setting. We freely use the concepts and notation of [3].

We denote a nodal Riemann surface by $(S, j, D, M)$, where $(S, j)$ is a compact Riemann surface, $D$ is the set of double points and $M$ is the set of marked points. As our curves have boundary, a nodal Riemann surface will have nodes of two types: boundary nodes, where both points are on the boundary, and interior nodal points, where both are in the interior. We do not consider mixed nodes. Also, we think of the boundary components as ordered, and so the set of marked points $M$ can be split as $M=M_{\text {int }} \cup M_{1} \cup$ $\ldots \cup M_{b}$, where $b \geq 0$ is the number of boundary components of the surface $S$, and where the marked points in $M_{\text {int }}$ are interior and the marked points in $M_{i}$ lie on the $i$ th boundary component. The genus of a nodal Riemann surface with boundary is the arithmetic genus of the topological surface obtained by filling each boundary component by a disc.

We define the signature of a nodal Riemann surface as the sequence $\sigma=\left(g, b ; n, m_{1}, \ldots, m_{b}\right)$, where $g$ is its genus, $b$ is the number of boundary components, $n$ is the number of interior marked points and $m_{i}$ is the number of marked points on the $i$ th boundary component.

The $\varepsilon$-thin part of every component of a stable nodal Riemann surface (with respect to its uniformizing metric) now consists of four types of domains: annuli of finite modulus around a short interior geodesic, annuli conformally equivalent to the punctured unit disc around each interior puncture, a rectangular region conformally equivalent to $[-1,1] \times(-L, L)$ around each short geodesic (minimal in its free homotopy class) connecting two boundary components and a region conformally equivalent to a punctured half-disc $D^{+} \backslash\{0\}$ near each boundary puncture.

A decoration (i.e., an orientation reversing orthogonal identification of the tangent planes at the two corresponding points) is required only at interior 
nodes, since at the boundary the choice of identification is fixed by matching the boundary directions. We denote a decorated nodal Riemann surface by $(S, j, D, M, r)$, where $r$ stands for the decoration.

We denote by $\overline{\mathcal{M}}_{\sigma}^{\$}$ the moduli space of connected decorated stable nodal Riemann surfaces with signature $\sigma$, equipped with the usual topology ([3], cf. also [14] for the nondecorated case). It is shown in $[\mathbf{3}]$ that, for each fixed signature $\sigma$, the space $\overline{\mathcal{M}}_{\sigma}^{\$}$ is a compact metric space which coincides with the closure of its subset $\mathcal{M}_{\sigma}$ of smooth marked Riemann surfaces with boundary of signature $\sigma$. In other words, every sequence of smooth stable marked Riemann surfaces $\left(S_{n}, j_{n}, M_{n}\right)$ of signature $\sigma$ has a subsequence which converges to a decorated nodal Riemann surface $(S, j, M, D, r)$ of the same signature.

Now consider $(X, L, \omega, J)$ as above. With the above setup for the domains, the definition of a nodal holomorphic curve of height 1 in $(X, L, J)$ is exactly the same as in [3, Section 8], except that we allow the domain to have boundary, which is required to be mapped to $L$. Similarly, we get the notion of a holomorphic building of height $\left(1 \mid k_{+}\right)$, and the notion of convergence. Fixing the signature $\sigma:=\left(g, b ; n, m_{1}, \ldots, m_{b}\right)$, we obtain the moduli space $\overline{\mathcal{M}}_{\sigma}(W, L, J)$ of stable holomorphic curves of that signature.

Now we can prove the Compactness Theorem 1.1 in the introduction, which we restate as follows.

Theorem 4.1. Let $(X, L, \omega, J)$ satisfy conditions $(\mathrm{X}),(\mathrm{Y}),(\mathrm{L}),(\mathrm{K})$ and (R). Then for any $E>0$ and for any fixed signature $\sigma=\left(g, b ; n, m_{1}, \ldots, m_{b}\right)$, the space $\overline{\mathcal{M}}_{\sigma}(X, L, J) \cap\{E(f) \leq E\}$ is compact.

Proof. The proof closely follows the strategy of the corresponding proof of Theorem 10.2. of [3]. Clearly, it is sufficient to establish sequential compactness for smooth curves (i.e., without nodes).

So let $f_{n}:\left(S_{n}, \partial S_{n}, j_{n}\right) \rightarrow(X, L, J)$ be a sequence of curves of fixed signature and uniformly bounded energy.

Step 1. After adding additional marked points if needed, we may assume that the underlying domains $\left(S_{n}, j_{n}, M_{n} \cup Z_{n}\right)$ of the $f_{n}$ are stable.

Now we want to argue that, by adding a finite set (with cardinality depending on the energy bound) of additional pairs of points, one obtains a new sequence of stable domains, denoted by $\left(S_{n}, j_{n}, M_{n} \cup Z_{n}\right)$, such that the new sequence satisfies the gradient bounds (4.1) and (4.2).

This is based on a bubbling analysis. Indeed, to achieve (4.1) one argues as in [3], producing finite energy planes or spheres that each take a minimal amount $\hbar>0$ of energy by Lemma 3.6.

So assume that (4.1) holds but (4.2) fails, i.e., there exists a sequence of points $z_{n} \in S_{n}$ such that $f_{n}\left(z_{n}\right) \in \mathcal{U}_{\varepsilon / 4}$ and $\left\|\nabla\left(\tau \circ f_{n}\right)\left(z_{n}\right)\right\| \cdot \rho\left(z_{n}\right) \rightarrow \infty$. After passing to a subsequence, we have one of the following two cases: 
(i) $\rho_{n}^{\prime}:=\frac{\rho\left(z_{n}\right)}{d\left(z_{n}, \partial S_{n}\right)} \leq C<\infty$, or

(ii) $\rho_{n}^{\prime} \rightarrow \infty$.

In case (i), we find holomorphic embeddings $\phi_{n}:(D, 0) \rightarrow\left(S_{n} \backslash\left(M_{n} \cup\right.\right.$ $\left.\left.Z_{n}\right), z_{n}\right)$ of the unit disk with

$$
\frac{1}{C^{\prime}} \rho_{n}^{\prime} \leq\left|\nabla \phi_{n}\right| \leq C^{\prime} \rho_{n}^{\prime}
$$

for some constant $C^{\prime}$, and in case (ii) we find points $\xi_{n} \in D^{+}$with $\xi_{n} \rightarrow 0$ and holomorphic embeddings $\phi_{n}:\left(D^{+}, D^{+} \cap \mathbb{R}, \xi_{n}\right) \rightarrow\left(S_{n} \backslash\left(M_{n} \cup Z_{n}\right), L, z_{n}\right)$ of the upper half disc satisfying the same bounds.

In both cases we can modify the sequence $\left(z_{n}\right)$, rescale $f_{n} \circ \phi_{n}$ as in $[\mathbf{3}$, Section 10.2.1] and apply Proposition 4.1 to obtain a $J$-holomorphic plane $f: \mathbb{C} \rightarrow X$ or half-plane $(\mathbb{H}, \mathbb{R}) \rightarrow(X, L)$ of finite energy. The map $f$ is either proper, or it extends to a holomorphic sphere or disc by Lemma 3.5. In either case, $f$ has area $E_{\omega}(f) \geq \hbar>0$ by Lemma 3.6. Hence adding a pair of marked points and repeating this process, we obtain a bound of the form (4.2) after finitely many steps.

Step 2. After passing to a subsequence, the domains $\left(S_{n}, M_{n}, Z_{n}\right)$ will converge to a decorated nodal Riemann surface with boundary $(S, j, M, Z, D, r)$. In Step 1 we have arranged for assumption (S2) to hold for our sequence, and using the energy bound we can arrange (S1) after passing to a subsequence. So, by Proposition 4.1, for a further subsequence we obtain $C_{\mathrm{loc}}^{\infty}$ convergence on each component of the complement of the pinching geodesics in $\left(S_{n}, j_{n}, M_{n}, Z_{n}\right)$ of the maps $f_{n}$ to some limiting map $f$ defined on the corresponding components of $(S, j, M, Z, D, r)$.

Step 3. Now we have to analyse the convergence in the thin part. Here, as in $[\mathbf{3}]$, one considers each type of component of the thin part separately. Annuli near interior marked points and near interior nodes are treated in detail in $[\mathbf{3}$, Section 10.2.3]. In the other two cases one proceeds analogously, with the following adaptions.

4.2.1. Behaviour near a boundary node. As in the case of interior nodes described in [3], boundary nodes appear as a result of degeneration of some component of the thin part of the $S_{n}$. The associated holomorphic strips $u_{n}=f_{n} \circ \phi_{n}$, obtained by precomposing with suitable uniformizations $\phi_{n}$ whose domains are longer and longer strips, have gradient bounds of the form

$$
\begin{aligned}
\left|\nabla u_{n}(z)\right| & \leq C \text { if } u_{n}(z) \notin \mathcal{U}_{\varepsilon / 4}, \\
\left|\nabla\left(\tau \circ u_{n}\right)(z)\right| & \leq C \text { if } u_{n}(z) \in \mathcal{U}_{\varepsilon} .
\end{aligned}
$$

This follows by the same argument as that for equation (35) in [3]. After passing to a subsequence, the areas $E_{\omega}\left(f_{n}\right)$ converge to either zero or some positive constant. 
First consider the case of zero limiting $E_{\omega}$-energy. If one of the asymptotics for the limit map $f$ in the adjacent thick parts of $S$ is a Reeb chord, one uses part (b) of Proposition 3.2 to conclude that the other asymptotic equals the same Reeb chord. If both adjacent asymptotics are points $p_{ \pm} \in L$ one uses Lemma 3.8 to conclude that $p_{+}=p_{-}$.

If the limiting $E_{\omega}$-energy of the strips is positive, in view of the gradient bounds (4.5) there can be no bubbling, and so the only possibility is breaking into a sequence of holomorphic strips. By Lemma 3.7, each nontrivial strip carries area at least $\hbar(E)$, so there can only be finitely many of them.

4.2.2. Behaviour near a boundary puncture. Here, the adjustments are similar in nature to the ones described for the previous case, and we omit the details.

After Step 3 is done, we have a subsequence $f_{n}$ of the original sequence of holomorphic curves converging to a limiting map $f$ defined on some nodal Riemann surface $(S, j, M, Z, D, r)$ such that $\lim E\left(f_{n}\right)=E(f)$.

Step 4. It remains to recover the level structure in the holomorphic building $f$ constructed above, and this is done exactly as in [3, Section 10.2.5].

Remark 4.2. Theorem 1.2 in $[\mathbf{1 3}]$ is a version of Gromov compactness for holomorphic curves in a symplectic manifold $X$ with boundaries on immersed Lagrangian submanifolds with mutually clean intersections. In order to discuss how it differs from our Theorem 1.1 we recall the notation from $[\mathbf{1 3}$, p. 81] (we restrict to the case of nonmoving boundary conditions since this is what we study in this paper).

The immersed boundary conditions are given by a finite collection of Lagrangian immersions $f_{i}: W_{i} \rightarrow X, i=1, \ldots, M$ with mutually clean intersections and self-intersections. The holomorphic curves $u_{n}: C_{n} \rightarrow X$, $n=1,2, \ldots$ of the sequence are assumed to be of the following form. The boundary $\partial C_{n}$ is subdivided into a fixed number of $\operatorname{arcs} \beta_{n, k}, k=1, \ldots, M$ and on any $\beta_{n, k}, u_{n}=f_{i} \circ u_{n, k}^{(b)}$ with continuous maps $u_{n, k}^{(b)}: \beta_{n, k} \rightarrow W_{i}$, for some $i$. This means that the number of switches where the map changes branches is a priori bounded by $M$. Contrary to that, in our Theorem 1.1 there is no a priori bound on the number of such switches. On the other hand $[\mathbf{1 3}]$ allows for more general almost complex structures.

\section{Proof of the Finiteness Theorem 1.2}

As before, we consider $(X, L, \omega, J)$ satisfying conditions $(\mathrm{X}),(\mathrm{Y}),(\mathrm{L}),(\mathrm{K})$ and $(\mathrm{R})$. Now we assume in addition that $(X, L, \omega=d \lambda)$ is exact with convex end, i.e., $\lambda$ is a positive contact form on $M$ which extends as a primitive of $\omega$ to $\bar{X}$.

For a holomorphic curve $f:(S, \partial S, j) \rightarrow(X, L, J)$ a switch is a point in $\partial S$ which is mapped to $K$. 
Now we can prove the Finiteness Theorem 1.2 in the introduction, which we restate for convenience.

Theorem 5.1. In the situation of Theorem 1.1, suppose in addition that $(X, L, \omega=d \lambda)$ is exact with convex end. Then for each $s \in \mathbb{N}$ and $C>0$ there exists a constant $\kappa(s, C) \in \mathbb{N}$ such that every holomorphic disc $f$ : $(\dot{D}, \partial \dot{D}, j) \rightarrow(X, L, J)$ with at most $s$ boundary punctures and energy $\leq C$ has at most $\kappa(s, C)$ switches.

Proof. We argue by contradiction. So assume there exists a sequence of holomorphic $\operatorname{discs} f:(\dot{D}, \partial \dot{D}, j) \rightarrow(X, L, J)$ with at most $s$ boundary punctures and energy $\leq C$ such that $f_{n}^{-1}(K) \cap \partial D$ contains at least $n$ points. After passing to a subsequence, we may assume that the number $s$ of boundary punctures and the ordered collection of asymptotic Reeb chords $\Gamma=\left(\gamma_{1}, \ldots, \gamma_{s}\right)$ is fixed in the sequence.

By Theorem 4.1 in the previous section, some subsequence of the $f_{n}$ converges to a stable holomorphic curve $f$ of some finite height $(1 \mid k)$, whose domain is a disc-like nodal Riemann surface $(S, j, Z, D, r)$ with $Z \subset \partial S$ of cardinality $s$. The convergence is in $C^{0}$ and in $C_{\mathrm{loc}}^{\infty}$ away from the punctures, the nodes and $f^{-1}(K) \cap \partial S$.

Consider a component $C$ of $S$ on which $f$ is nonconstant. We claim that in this case $f^{-1}(K) \cap \partial C$ is finite. To see this, suppose otherwise. Since $f$ tends to infinity near the boundary punctures the set $f^{-1}(K) \cap \partial C$ avoids a neighbourhood of the punctures and thus has a limit point $p \in \partial C$. Pick a neighbourhood $S_{p}$ of $p$ which is mapped into a neighbourhood as in condition (K) on which we have holomorphic coordinates mapping the branches of $L$ to $\mathbb{R}^{n}$ and $\mathbb{R}^{k} \times \mathrm{i} \mathbb{R}^{n-k}$. Consider for $\ell=k+1, \ldots, n$ the holomorphic map $\nu_{\ell} \circ f: S_{p} \rightarrow \mathbb{C}$, where $\nu_{\ell}: \mathbb{C}^{n} \rightarrow \mathbb{C}$ is the projection onto the $\ell$ th $\mathbb{C}$ factor in these coordinates. Since $\nu_{\ell} \circ f$ has infinitely many zeros in $S_{p}$, Lemma 2.5 implies that it vanishes identically, so $f\left(S_{p}\right) \subset K^{\mathbb{C}}$, where $K^{\mathbb{C}} \subset$ $\mathcal{U}_{\varepsilon}$ is the complexification of $K$ in condition $(\mathrm{K})$. By unique continuation, the component of $p$ in $\left.f^{-1}\left(\mathcal{U}_{\varepsilon}\right)\right)$ is mapped into $K^{\mathbb{C}}$, so in particular the (connected) boundary of $C$ is mapped entirely into $K$. But since $L$ is exact, the boundary of a nonconstant component must contain at least one positive puncture. This contradiction completes the proof of the claim.

It follows that $f^{-1}(K) \cap \partial S$ consists of finitely many points and finitely many components on which $f$ takes a constant value on $K$. Pick disjoint compact sets $S_{1}, \ldots, S_{r}$ with piecewise smooth boundary such that $f^{-1}(K) \cap$ $\partial S \subset \cup_{i}$ int $S_{i}$ and for each $S_{i}$ one of the following holds:

(a) $S_{i}$ contains precisely one point of $f^{-1}(K) \cap \partial S$ and no nodes, or

(b) $S_{i}$ contains precisely one connected union of components on which $f$ takes a constant value on $K$.

Moreover, we may assume that each $S_{i}$ is mapped into the neighbourhood $\mathcal{U}_{\varepsilon}$ of $K$ in condition $(\mathrm{K})$. Note that for $n \geq N$ sufficiently large we 
have $f_{n}\left(\partial D \backslash \cup_{i} S_{i}\right) \cap K=\varnothing$ by the $C^{0}$-convergence on compact sets. Since $f_{n}^{-1}(K) \cap \partial D$ contains at least $n$ points, it follows that in some $S_{i}$ the map $f_{n}$ has at least $n / r$ points of $\partial D$ mapping to $K$.

Suppose first that this $S_{i}$ is of type (a). Then $f_{n} \rightarrow f$ in $C^{\infty}$ on $S_{i}$ and (composing as above with projections to $\mathbb{C}$ ) Lemma 2.5 implies that $f$ maps $S_{i}$ into $K^{\mathbb{C}}$. As above, this yields a contradiction.

Finally, suppose that $S_{i}$ is of type (b). Then we argue as in the proof of Lemma 2.5: By Lemma 2.3, the winding number of $f_{n}$ over $\Gamma_{i}:=\partial S_{i} \backslash\left(S_{i} \cap\right.$ $\partial S)$ satisfies

$$
w\left(f_{n}, \Gamma_{i}\right) \geq \frac{n}{4 r} \stackrel{n \rightarrow \infty}{\longrightarrow} \infty .
$$

On the other hand, the smooth convergence $f_{n} \rightarrow f$ on $\Gamma_{i}$ implies

$$
w\left(f_{n}, \Gamma_{i}\right) \stackrel{n \rightarrow \infty}{\longrightarrow} w\left(f, \Gamma_{i}\right)<\infty .
$$

This contradiction completes the proof of the Finiteness Theorem.

Corollary 5.1. In the situation described above, for every ordered collection of Reeb chords $\Gamma=\left(\gamma_{1}, \ldots, \gamma_{s}\right)$, there exists a constant $\kappa=\kappa(\Gamma)$ with the following property. If $Z \subset \partial D^{2}$ has cardinality $s$ and $f:\left(D^{2} \backslash Z, \partial D^{2} \backslash Z\right) \rightarrow$ $\left(T^{*} \mathbb{R}^{3}, L\right)$ is a J-holomorphic disc with asymptotics $\Gamma$, then $f^{-1}(K) \cap \partial D^{2}$ contains at most $\kappa$ points. In particular, $f$ has at most $\kappa$ switches.

\section{Appendix A. Dimensions of moduli spaces}

Consider a quadruple $(X, L, \omega, J)$ satisfying conditions $(\mathrm{X}),(\mathrm{Y}),(\mathrm{L}),(\mathrm{K})$ and $(\mathrm{R})$ in Section 3. In this appendix we give the dimension formula for moduli spaces of holomorphic curves in $X$ with smooth boundary on $L$, interior punctures asymptotic to closed Reeb orbits, boundary punctures asymptotic to Reeb cords and Lagrangian intersection punctures, i.e., boundary punctures asymptotic to the clean self-intersection $K$. Before proving the formula we introduce the (topological) data needed to state it.

Consider a Lagrangian intersection puncture mapping to a point $k$ in a component $K_{d}$ of $K$, where $\operatorname{dim}\left(K_{d}\right)=n-d$. We restate the relevant part of condition $(\mathrm{K})$ as follows:

(t0) Near $k$ there exist local holomorphic coordinates $\mathbb{C}^{n-d} \times \mathbb{C}^{d}$ in which $L$ corresponds to $\mathbb{R}^{n-d} \times\left(\mathbb{R}^{d} \cup i \mathbb{R}^{d}\right)$, and $T^{*} K$ corresponds to $\mathbb{C}^{n-d} \times\{0\}$.

Assume that $f:(S, \partial S) \rightarrow(X, L)$ is a holomorphic map with a Lagrangian intersection puncture. Pick a local coordinate $z$ in upper half plane $\mathbb{H}$ on the source $S$, where the Lagrangian intersection puncture corresponds to $0 \in \mathbb{H}$, and local holomorphic coordinates $\mathbb{C}^{n-d} \times \mathbb{C}^{d}$ around $f(0)$ in the target as in $(\mathrm{t} 0)$. In these coordinates $f$ is expressed as

$$
f(z)=\left(f_{1}(z), f_{2}(z)\right) \in \mathbb{C}^{n-d} \times \mathbb{C}^{d},
$$


where $f_{1}$ maps $\mathbb{R}$ to $\mathbb{R}^{n-d}$, and $f_{2}$ maps $\mathbb{R}_{ \pm}$to $\mathbb{R}^{d}$ or $i \mathbb{R}^{d}$. It follows that $f_{1}, f_{2}$ have unique power series expansions of the form

$$
f_{1}(z)=\sum_{j=0}^{\infty} a_{j} z^{j}, \quad f_{2}(z)=\sum_{j=0}^{\infty} c_{j} z^{j+w},
$$

where $a_{j} \in \mathbb{R}^{n-d}$ for all $j$, either $c_{j} \in \mathbb{R}^{d}$ for all $j$ or $c_{j} \in \mathrm{i} \mathbb{R}^{d}$ for all $j, c_{0} \neq 0$, and $w$ is either a positive half integer (if the map $f$ switches local sheets of $L$ at $k$ ) or a positive integer (if $f$ remains on one sheet). We call $w$ the asymptotic winding number of $f$ at the Lagrangian intersection puncture $k$. (This notion is clearly independent of the choices involved in its definition.)

Assume that the holomorphic map $f:(S, \partial S) \rightarrow(X, L)$ has

- $p$ positive interior punctures at Reeb orbits $\gamma_{1}, \ldots, \gamma_{p}$,

- $q$ negative interior punctures at Reeb orbits $\beta_{1}, \ldots, \beta_{q}$,

- $s$ positive boundary punctures at Reeb chords $c_{1}, \ldots, c_{s}$,

- $t$ negative boundary punctures at Reeb chords $b_{1}, \ldots, b_{t}$, and

- $l$ Lagrangian intersection punctures on the boundary mapping to clean self intersection components $K_{d_{1}}, \ldots, K_{d_{l}}$ with asymptotic winding numbers $w_{1}, \ldots, w_{l}$, respectively, where $\operatorname{dim}\left(K_{d_{j}}\right)=\left(n-d_{j}\right)$, $j=1, \ldots, l$.

We trivialize $T X$ along parts of the map $f$ as follows:

(t1) Fix complex trivializations $Z_{\gamma}$ of the contact planes in the convex end of $X$ along all Reeb orbits $\gamma \in\left\{\gamma_{1}, \ldots, \gamma_{p}\right\}$.

(t2) Fix complex trivializations $Z_{\beta}$ of the contact planes in the concave end of $X$ along all Reeb orbits $\beta \in\left\{\beta_{1}, \ldots, \beta_{q}\right\}$.

If $\alpha \in\left\{\gamma_{1}, \ldots, \gamma_{p}\right\}$ or $\alpha \in\left\{\beta_{1}, \ldots, \beta_{q}\right\}$ then the linearized Reeb flow induces a one-parameter family of symplectomorphisms $\Phi_{t}: \xi_{\alpha(0)} \rightarrow \xi_{\alpha(t)}$, where $\xi_{\alpha(t)}$ is the contact hyperplane at $\alpha(t), t \in[0, T]$. Using the trivialization $Z_{\alpha}$ from (t1) or (t2), we view $\Phi_{t}$ as a path of symplectomorphisms $\Phi_{t}^{Z_{\alpha}}: \mathbb{C}^{n-1} \rightarrow$ $\mathbb{C}^{n-1}$. Write

$$
\mu_{\mathrm{CZ}}\left(\alpha, Z_{\alpha}\right)
$$

for the Conley-Zehnder index of the path $\Phi_{t}^{Z_{\alpha}}, 0 \leq t \leq T$ (see [8]).

Remark A.1 (cf. [8]). The Conley-Zehnder index of a path $\Psi_{t}: \mathbb{C}^{m} \rightarrow$ $\mathbb{C}^{m}, 0 \leq t \leq 1$ is the Maslov index of the path of Lagrangian planes in $\mathbb{C}^{m} \oplus \mathbb{C}^{m}$ corresponding to the graph of $\Psi_{t}$. The Maslov index of a path $L_{t}, 0 \leq t \leq 1$, of Lagrangian planes in $\mathbb{C}^{k}$ equals $\langle\mu,[\hat{L}]\rangle-\frac{k}{2}$, where $\mu$ is the Maslov class and where $\hat{L}$ is the loop of Lagrangian planes obtained by closing $L$ by a positive rotation taking $L_{1}$ and $L_{0}$. Here a positive rotation is defined as follows. Two Lagrangian subspaces $V_{0}$ and $V_{1}$ in $\mathbb{C}^{k}$ defines a decomposition $W=W^{1} \oplus \ldots \oplus W^{r}$ into orthogonal subspaces and a 
complex angle $\left(\theta_{1}, \ldots, \theta_{r}\right), 0 \leq \theta_{1}<\theta_{2}<\ldots<\theta_{r}<\pi$ as follows. Let $\theta_{1}$ be the smallest number in $[0, \pi)$ such that

$$
\operatorname{dim}\left(\left(\mathrm{e}^{\mathrm{i} \theta_{1}} \cdot V_{0}\right) \cap V_{1}\right) \geq 1 .
$$

Let $W^{1} \subset \mathbb{C}^{k}$ be the complex subspace generated by $\left(\mathrm{e}^{\mathrm{i} \theta_{1}} \cdot V_{0}\right) \cap V_{1}$ and let $W^{\prime}$ be its orthogonal complement. Then $V_{0}^{\prime}=W^{\prime} \cap\left(\mathrm{e}^{\mathrm{i} \theta_{1}} \cdot V_{0}\right)$ and $V_{1}^{\prime}=W^{\prime} \cap V_{1}$ are Lagrangian subspaces. Let $\theta_{1}^{\prime}$ be the smallest number in $(0, \pi)$ such that

$$
\operatorname{dim}\left(\left(\mathrm{e}^{\mathrm{i} \theta_{1}^{\prime}} \cdot V_{0}^{\prime}\right) \cap V_{1}^{\prime}\right) \geq 1 .
$$

Let $\theta_{2}=\theta_{1}^{\prime}+\theta_{1}$ and let $W^{2} \subset W^{\prime} \subset W$ be the complex subspace generated by $\left(\mathrm{e}^{\mathrm{i} \theta_{1}^{\prime}} \cdot V_{0}^{\prime}\right) \cap V_{1}^{\prime}$. Repeating this construction we get a decomposition and complex angles as claimed.

The positive rotation taking $V_{0}$ to $V_{1}$ is the one-parameter family of linear transformations which acts by multiplication by $\mathrm{e}^{\mathrm{i} \theta_{j} t}, t \in[0,1]$ on $W^{j}, j=$ $1, \ldots, r$. The negative rotation taking $V_{0}$ to $V_{1}$ acts by multiplication by $\mathrm{e}^{-\mathrm{i}\left(\pi-\theta_{j}\right) t}, t \in[0,1]$ on $W^{j}, j=1, \ldots, r$.

(t3) Fix complex trivializations $Z_{c}$ of the contact planes along all Reeb chords $c \in\left\{c_{1}, \ldots, c_{s}\right\}$ of the Legendrian submanifold in the convex end which have the property that the linearized Reeb flow along the chord $c$ expressed in $Z_{c}$ is constantly equal to the identity.

(t4) Fix complex trivializations $Z_{b}$ of the contact planes along all Reeb chords $b \in\left\{b_{1}, \ldots, b_{t}\right\}$ of the Legendrian submanifold in the concave end which have the property that the linearized Reeb flow along the chord $b$ expressed in $Z_{b}$ is constantly equal to the identity.

Completing these trivializations with a vector field in the symplectization direction, we get trivializations of $T X$ along any Reeb orbit and along any Reeb chord appearing as asymptotic data for $f$.

(t5) Fix complex trivializations $Z_{C}$ of $f^{*} T X$ along each component $C$ of the complement of the punctures in $\partial S$ with the following properties. If an endpoint of $C$ is a Reeb chord puncture at a Reeb chord $a$ then $Z_{C}=Z_{a}$ at the corresponding Reeb chord endpoint in some neighborhood of the endpoint of $C$. If a Lagrangian intersection puncture is the common endpoint of boundary components $C$ and $C^{\prime}$ then $Z_{C}=Z_{C^{\prime}}$ at the common endpoint.

Choices (t1) and (t2) give trivializations of $f^{*} T X$ near each interior puncture in $S$ and choices (t3)-(t5) give a trivializations $Z_{\partial_{j} f}$ of $f^{*} T X$ along the $j$ th component $C_{j}$ of the boundary $\partial S$, where we think of punctures as marked points so that $\partial S$ becomes a closed one-manifold. Let

$$
c_{1}^{\mathrm{rel}}\left(u^{*}(T X) ; Z_{\partial f} ; Z_{\gamma_{1}}, \ldots, Z_{\gamma_{p}} ; Z_{\beta_{1}}, \ldots, Z_{\beta_{q}}\right)
$$

denote the obstruction to extending this trivialization over $S$. Here we think of the obstruction as the number arising from evaluating the obstruction 
class on the orientation class of $\left(S_{0}, \partial S_{0}\right)$, where $S_{0}$ is the surface obtained from $S$ by removing small open discs around all its interior punctures and where the bundle is trivialized along $\partial S_{0}$.

Let $\Lambda$ denote a Legendrian submanifold at one of the ends of $X$ and let $a \in\left\{c_{1}, \ldots, c_{s}\right\}$ or $a \in\left\{b_{1}, \ldots, b_{t}\right\}$ be a Reeb chord of $\Lambda$. Let $a^{-}$denote the endpoint of $a$ where the Reeb vector field points into $a$, and let $a^{+}$denote the other endpoint of $a$. The image of the tangent space $T_{a^{-}} \Lambda$ under the linearized Reeb flow along $a$ is a Lagrangian plane $\left(T_{a^{-}} \Lambda\right)^{\prime} \subset \xi_{a^{+}}$, where $\xi_{y}$ denotes the contact plane at $y$. Assume that $a$ is generic in the sense that the two Lagrangian subspaces $\left(T_{a^{-}} \Lambda\right)^{\prime}$ and $T_{a^{+}} \Lambda$ of $\xi_{a^{+}}$intersect transversely (after small perturbation, all Reeb chords are generic). Let

$$
R_{a^{+}}^{\text {neg }}\left(a^{-}, a^{+}\right): \xi_{a^{+}} \rightarrow \xi_{a^{+}}
$$

denote the rotation in $\xi_{a^{+}}$in the negative direction which takes $\left(T_{a^{-}} \Lambda\right)^{\prime}$ to $T_{a^{+}} \Lambda$. Let

$$
R_{a^{-}}^{\mathrm{neg}}\left(a^{+}, a^{-}\right): \xi_{a^{-}} \rightarrow \xi_{a^{-}}
$$

be defined similarly, rotating the image $\left(T_{a^{+}} \Lambda\right)^{\prime}$ of $T_{a^{+}} \Lambda$, under the backwards linearized Reeb flow along $a$, in the negative direction in $\xi_{a^{-}}$to $T_{a^{-}} \Lambda$.

Let $C_{j}^{\prime}$ denote the complement of the punctures in the $j$ th component $C_{j} \subset \partial S$. Then the tangent planes to $L$ along $f\left(C_{j}^{\prime}\right)$ expressed in the trivializations $Z_{\partial_{j} f}$ constitute a collection of paths of Lagrangian planes in $\mathbb{C}^{n}$. We close these paths to a loop as follows:

(t3') The tangent planes of $L=\Lambda \times \mathbb{R}$ at endpoints of a Reeb chord $c \in$ $\left\{c_{1}, \ldots, c_{s}\right\}$ are connected by the product of the linearized Reeb flow along $c$ in $\xi$ and the identity in the symplectization direction, followed by the path

$$
R_{c^{+}}^{\text {neg }}\left(c^{-}, c^{+}\right)\left(\left(T_{c^{-}} \Lambda\right)^{\prime}\right) \oplus \mathbb{R} \subset \xi_{c^{+}} \oplus \mathbb{C} .
$$

(t4 $4^{\prime}$ ) The tangent planes of $L=\Lambda \times \mathbb{R}$ at endpoints of a Reeb chord $b \in\left\{b_{1}, \ldots, b_{t}\right\}$ are connected by the backwards linearized Reeb flow along $b$ in $\xi$ and the identity in the symplectization direction, followed by the path

$$
R_{b^{-}}^{\text {neg }}\left(b^{+}, b^{-}\right)\left(\left(T_{b^{+}} \Lambda\right)^{\prime}\right) \oplus \mathbb{R} \subset \xi_{b^{-}} \oplus \mathbb{C} .
$$

$\left(\mathrm{t}^{\prime}\right)$ The tangent planes at a Lagrangian intersection puncture mapping to $K_{d} \in\left\{K_{d_{1}}, \ldots, K_{d_{l}}\right\}$ of asymptotic winding number $w$ correspond to the planes $\mathbb{R}^{n-d} \times \mathbb{R}^{d}$ or $\mathbb{R}^{n-d} \times \mathrm{i} \mathbb{R}^{d}$ in the coordinates (t0). Connect these planes by multiplying the tangent plane of the boundary component oriented toward the puncture with the matrix

$$
\left(\begin{array}{cc}
1 & 0 \\
0 & \mathrm{e}^{-s w \pi \mathrm{i}}
\end{array}\right), \quad 0 \leq s \leq 1
$$

in the local $\mathbb{C}^{n-d} \times \mathbb{C}^{d}$-coordinates of (t0). 
Define

$$
\mu\left(\partial_{j} f, Z_{\partial_{j} f}\right)
$$

as the Maslov index of the loop of Lagrangian subspaces in $\mathbb{C}^{n}$ which corresponds to the $j$ th boundary component of $S$ and which is constructed by closing the paths of Lagrangian planes as described in $\left(\mathrm{t} 3^{\prime}\right),\left(\mathrm{t} 4^{\prime}\right)$ and $\left(\mathrm{t} 0^{\prime}\right)$.

Let $\mathcal{M}(f)$ denote the moduli space of holomorphic curves in $X$ with boundary on $L$, with punctures at Reeb orbits, Reeb chords and at Lagrangian self-intersection components as described above, which have the same additional structure as $f$ (i.e., asymptotics and Lagrangian intersection punctures, including asymptotic windings), have domain diffeomorphic to $(S, \partial S)$, and which are homotopic to $f$ through (continuous) maps respecting the additional structure. Recall that $\operatorname{dim}(X)=2 n$, let $g$ denote the genus of $S$, and let $r$ denote the number of boundary components of $\partial S$.

Theorem A.1. With the notation from (A.2), (A.3) and (A.5), the formal dimension of $\mathcal{M}(f)$ is given by

$$
\begin{aligned}
\operatorname{dim}(\mathcal{M}(f))= & (n-3)(2-2 g-r)+(s+t+l) \\
& +\sum_{j=1}^{p}\left(\mu_{\mathrm{CZ}}\left(\gamma_{j}, Z_{\gamma_{j}}\right)-(n-3)\right) \\
& -\sum_{j=1}^{q}\left(\mu_{\mathrm{CZ}}\left(\beta_{j}, Z_{\beta_{j}}\right)+(n-3)\right) \\
& +\sum_{j=1}^{r} \mu\left(\partial_{j} f, Z_{\partial_{j} f}\right) \\
& +2 c_{1}^{\mathrm{rel}}\left(u^{*}(T X) ; Z_{\partial f} ; Z_{\gamma_{1}}, \ldots, Z_{\gamma_{p}} ; Z_{\beta_{1}}, \ldots, Z_{\beta_{q}}\right) .
\end{aligned}
$$

Remark A.2. As mentioned in Section 1, it follows from Theorem A.1 that the contribution from a Lagrangian intersection puncture mapping to a codimension $d$ clean intersection component with asymptotic winding number $w$ equals $1-w d$. Here 1 is the contribution to $l$ and $-w d$ is the contribution to the Maslov index from the rotation in (A.4).

Proof. We consider first the case when there are no Lagrangian intersection punctures. The formal dimension of $\mathcal{M}(f)$ equals the Fredholm index of the linearization of the $\bar{\partial}_{J}$-equation at $f$. The source space of this operator splits into the direct sum of an infinite-dimensional functional analytic space of vector fields along $f$ and the tangent space of the space of conformal structures of the domain $S$ of $f$. We denote the restriction of the linearized $\bar{\partial}_{J}$-operator to the space of vector fields by $\bar{\partial}_{\mathrm{vf}}$.

The index of $\bar{\partial}_{\mathrm{vf}}$ remains constant as the operator is deformed through Fredholm operators. Consider the symplectization direction in $f^{*} T X$ near 
any boundary puncture in $S$. The boundary condition in this direction is degenerate. In order to describe a neighbourhood of the map $f$ in a functional analytic setting (e.g., a polyfold neighbourhood of $f$ ), one would use a Sobolev space with small positive exponential weights at the punctures and augment that space by one cut-off solution corresponding to translations in the $\mathbb{R}$-direction for each puncture. (With notation as above, if $\theta_{m}$ and $\theta_{M}$ denotes the smallest and largest complex angles, respectively, of $\left(T_{a^{-}} \Lambda\right)^{\prime}$ and $T_{a^{+}} \Lambda$ over all Reeb chords $a \in\left\{c_{1}, \ldots, c_{p}\right\} \cup\left\{b_{1}, \ldots, b_{t}\right\}$ then the weight being small means that it is smaller than $\min \left\{\theta_{m}, \pi-\theta_{M}\right\}$.) However, for index purposes, this is equivalent to forgetting the cut-off solution and changing the weight to a small negative exponential weight. We will work in the setting of small negative exponential weight without auxiliary solutions below.

The first deformation of $\partial_{\mathrm{vf}}$ will change the $\xi$-directions of the boundary condition near boundary punctures so that they look like the symplectization direction. Consider the boundary condition at a boundary puncture mapping to a Reeb chord $a$. We deform it as follows. Rotate the image of the tangent space $\left(T_{a^{ \pm}} \Lambda\right)^{\prime}$ at the endpoint $a^{\mp}$ of a boundary arc of $f$ under the linearized Reeb flow along $a$ (forwards or backwards according to the sign of the boundary puncture) in the negative direction to $T_{a^{\mp}} \Lambda$ and simultaneously change the weight at this puncture in the $\xi$-directions from its initial value $1=\mathrm{e}^{0}$ to a small negative exponential weight. It is straightforward to check that this gives a path of Fredholm operators, compare [8, Proposition 6.14]. Denote the operator at the endpoint of this path $\bar{\partial}_{\mathrm{vf}}^{\prime}$. Consider the surface $\hat{S}$ which is $S$ with boundary punctures erased. Since the change of coordinates taking a neighbourhood $[0, \infty) \times[0,1]$ of a puncture in $S$ to a neighborhood of $0 \in \mathbb{H}$ of the corresponding point in $\hat{S}$ is $w \mapsto \mathrm{e}^{-\pi w}$ it follows that the index of the operator $\bar{\partial}_{\mathrm{vf}}^{\prime}$ on $S$ equals the index of the $\bar{\partial}$-operator on the surface $\hat{S}$, with boundary condition naturally induced from the boundary condition of $\bar{\partial}_{\mathrm{vf}}^{\prime}$, see [8, Proposition 6.13]. We denote this operator on $\hat{S}$ by $\bar{\partial}_{\hat{S}}$. By definition, in the trivialization $Z_{\left(\partial_{j} f\right)}$ along the $j$ th boundary component of $\hat{S}$, the Lagrangian boundary condition of $\bar{\partial}_{\hat{S}}$ has Maslov index $\mu\left(\partial_{j} f ; Z_{\partial_{j} f}\right)$.

We next consider interior punctures. Also here, the asymptotic operator is degenerate in the symplectization direction. As in the case of boundary punctures, one would use small positive exponential weights and cut-off solutions to define functional analytic neighbourhoods, but in order to compute the index we might as well use small negative exponential weights and no cut-off solutions. (Here small refers to small when compared to the distance between the eigenvalues of the linearized return maps and 1.)

Fix capping spheres of all Reeb orbits at interior punctures. A capping sphere of a positive (negative) puncture where $f$ is asymptotic to a Reeb 
orbit $\alpha$ is a once punctured sphere with a trivial $\mathbb{C}^{n-1} \oplus \mathbb{C}$-bundle over it with trivialization which extends the trivialization $Z_{\alpha}$ given near the puncture and with a $\bar{\partial}$-operator with the asymptotics of a negative (positive) puncture at $\alpha$ in the $\mathbb{C}^{n-1}$-direction and with trivial asymptotics and small positive exponential weight in the symplectization direction corresponding to $\mathbb{C}$. Thus, the capping operator $\bar{\partial}_{\alpha}^{+}$of a positive puncture at $\alpha$ has index

$$
\operatorname{ind}\left(\bar{\partial}_{\alpha}^{+}\right)=(n-1)-\mu_{\mathrm{CZ}}\left(\alpha, Z_{\alpha}\right),
$$

and the capping operator $\bar{\partial}_{\alpha}^{-}$of a negative puncture at $\alpha$ has index

$$
\operatorname{ind}\left(\bar{\partial}_{\alpha}^{-}\right)=(n-1)+\mu_{\mathrm{CZ}}\left(\alpha, Z_{\alpha}\right),
$$

see $[\mathbf{4}, \mathbf{1 7}]$. A well-known argument shows that the index is additive under linear gluing of operators. We make one remark concerning this result in the present setup: the symplectization $\mathbb{C}$-component of the operator on a gluing neck limits to the standard operator on the infinite cylinder with positive exponential weight at one end and negative exponential weight at the other. This Fredholm operator is invertible and the usual linear gluing argument applies.

The result of gluing the capping spheres at the punctures of $\hat{S}$ and the capping operators to the operator $\bar{\partial}_{\hat{S}}$ is a $\bar{\partial}$-operator $\bar{\partial}_{\bar{S}}$ on a surface $\bar{S}$ of genus $g$ with $r$ boundary components and a Lagrangian boundary condition along each boundary component. The complex bundle over $\bar{S}$ comes equipped with a trivialization $Z$ near its boundary. Let $\mu(\partial \bar{S}, Z)$ denote the total Maslov index of the Lagrangian boundary condition of the boundary measured with respect to the trivialization $Z$ and let $c_{1}^{\text {rel }}(Z)$ denote the relative Chern class which is the obstruction to extending $Z$ from $\partial \bar{S}$ to all of $\bar{S}$. Doubling $\bar{S}$ as well as the operator $\bar{\partial}_{\bar{S}}$ over the boundary of $\bar{S}$ and applying the Riemann-Roch formula in combination with complex conjugation gives

$$
\operatorname{ind}\left(\bar{\partial}_{\bar{S}}\right)=n(2-2 g-r)+\mu(\partial \bar{S}, Z)+2 c_{1}^{\mathrm{rel}}(Z) .
$$

Additivity of the index then gives

$$
\begin{aligned}
\operatorname{ind}\left(\bar{\partial}_{\mathrm{vf}}\right)= & \operatorname{ind}\left(\bar{\partial}_{\hat{S}}\right) \\
= & n(2-2 g-r)+\sum_{j=1}^{p}\left(\mu_{\mathrm{CZ}}\left(\gamma_{j}, Z_{\gamma_{j}}\right)-(n-1)\right) \\
& -\sum_{j=1}^{q}\left(\mu_{\mathrm{CZ}}\left(\beta_{j}, Z_{\beta_{j}}\right)+(n-1)\right)+\mu(\partial \bar{S}, Z)+2 c_{1}^{\mathrm{rel}}(Z) \\
= & n(2-2 g-r)+\sum_{j=1}^{p}\left(\mu_{\mathrm{CZ}}\left(\gamma_{j}, Z_{\gamma_{j}}\right)-(n-1)\right)
\end{aligned}
$$




$$
\begin{aligned}
& -\sum_{j=1}^{q}\left(\mu_{\mathrm{CZ}}\left(\beta_{j}, Z_{\beta_{j}}\right)+(n-1)\right)+\sum_{j=1}^{r} \mu\left(\partial_{j} f, Z_{\partial_{j} f}\right) \\
& +2 c_{1}^{\mathrm{rel}}\left(f^{*}(T X) ; Z_{\partial f} ; Z_{\gamma_{1}}, \ldots, Z_{\gamma_{p}} ; Z_{\beta_{1}}, \ldots, Z_{\beta_{q}}\right) .
\end{aligned}
$$

In order to compute the dimension it remains only to compute the dimension $\operatorname{dim}(\mathcal{T})$ of the space $\mathcal{T}$ of conformal structures on $S$. Doubling a surface with $r$ boundary components in a similar way as above, studying the $\bar{\partial}$-equation for vector fields along the surface which are tangent to the boundary along the boundary, and noting that each interior puncture adds two degrees of freedom and each boundary puncture adds one degree of freedom, we find that the dimension of the space of conformal structures on $S$ equals

$$
\operatorname{dim}(\mathcal{T})=3 r+(s+t)+2(p+q)-6+6 g .
$$

We thus have

$$
\begin{aligned}
\operatorname{dim}(\mathcal{M}(f))= & \operatorname{ind}\left(\bar{\partial}_{\mathrm{vf}}\right)+\operatorname{dim}(\mathcal{T}) \\
= & (n-3)(2-2 g-r)+(s+t) \\
& +\sum_{j=1}^{p}\left(\mu_{\mathrm{CZ}}\left(\gamma_{j}, Z_{\gamma_{j}}\right)-(n-3)\right)-\sum_{j=1}^{q}\left(\mu_{\mathrm{CZ}}\left(\beta_{j}, Z_{\beta_{j}}\right)+(n-3)\right) \\
& +\sum_{j=1}^{r} \mu\left(\partial_{j} f, Z_{\partial_{j} f}\right) \\
& +2 c_{1}^{\mathrm{rel}}\left(f^{*}(T X) ; Z_{\partial f} ; Z_{\gamma_{1}}, \ldots, Z_{\gamma_{p}} ; Z_{\beta_{1}}, \ldots, Z_{\beta_{q}}\right),
\end{aligned}
$$

finishing the proof in the case when there are no Lagrangian intersection punctures.

Consider next the case when there are Lagrangian intersection punctures. In order to define a functional analytic neighbourhood of a map $f$ with such punctures of given asymptotic winding number $w$ mapping to a codimension $d$ component of the clean intersection, we puncture the boundary of $S$ and identify a neighbourhood of the puncture in the domain with $[0, \infty) \times[0,1]$ by the change of variables $z=\mathrm{e}^{-\pi w}, w=\tau+i t \in[0, \infty) \times[0,1]$. Then the Taylor expansion (A.1) gives

$$
f_{1}(\tau+\mathrm{i} t)=\sum_{j=0}^{\infty} a_{j} \mathrm{e}^{-\pi j(\tau+\mathrm{i} t)}, \quad f_{2}(\tau+\mathrm{i} t)=\sum_{j=0}^{\infty} c_{j} \mathrm{e}^{-\pi(j+w)(\tau+\mathrm{i} t)} .
$$

It follows that a neighbourhood can be modelled on a Sobolev space with positive exponential weight $\mathrm{e}^{\left(w-\frac{1}{100}\right) \tau}$ augmented by the space of cut off solutions spanned by

$$
\psi \cdot a_{0}, \psi \cdot a_{1} \mathrm{e}^{-\pi(\tau+\mathrm{i} t)}, \ldots, \psi \cdot a_{v} \mathrm{e}^{-\pi v(\tau+\mathrm{i} t)},
$$


where $\psi$ is a cut off function on $[0, \infty) \times[0,1]$, where $v$ is the largest integer smaller than $w-\frac{1}{100}$, and where $a_{j} \in \mathbb{R}^{n-d}$. (This augmentation space has dimension $(n-d)(v+1)$.)

At Lagrangian intersection punctures where the map switches local sheets of $L$, we observe that, as for Reeb chords above, we can close up the boundary condition at a Lagrangian intersection puncture by rotating $-\frac{\pi}{2}$ in $\mathbb{C}^{d}$, keeping the weight, and obtain a family of Fredholm operators.

Finally, we interpret the above weights in terms of the closed up boundary condition along $\partial \hat{S}$. In the source we use the change of variables $z=\mathrm{e}^{-\pi w}$, $w=\tau+\mathrm{i} t \in S$ and $z \in \hat{S}$ as above. We conclude that an exponential weight in $w$-coordinates of magnitude $k^{\prime} \pi$, where $k-1<k^{\prime}<k$ for an integer $k \geq 1$, corresponds to the condition that the sections of $f^{*} T X$ and their first $k-1$ derivatives vanishes at 0 in the $z$-coordinates. Thus the dimension formula is obtained by applying the formula above to the boundary condition obtained by closing up the Lagrangian boundary conditions at each Lagrangian intersection puncture with a minimal negative rotation (i.e., if the map switches sheets at the puncture we rotate by $-\frac{\pi}{2}$ in the $C^{d}$-factor

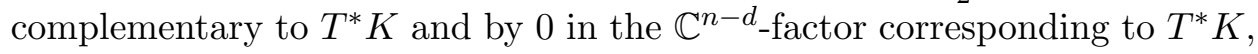
and if the map does not switch sheets we rotate by 0 in both factors) and adding

$$
1-w^{\prime} d
$$

for each Lagrangian intersection puncture, where $w^{\prime}$ is the largest integer smaller than $w$. Here 1 comes from the increase in the dimension of the space of conformal structures $\mathcal{T}$. The theorem then follows by definition of the close up at Lagrangian intersection punctures, see (A.4).

\section{References}

[1] C. Abbas, Finite energy surfaces and the chord problem, Duke Math. J. 96(2) (1999), 241-316.

[2] A. Abbondandolo and M. Schwarz, Floer homology of cotangent bundles and the loop product, 2009, arXiv:0810.1995.

[3] F. Bourgeois, Y. Eliashberg, H. Hofer, K. Wysocki and E. Zehnder, Compactness results in symplectic field theory, Geom. Topol. 7 (2003), 799-888.

[4] F. Bourgeois and K. Mohnke, Coherent orientations in symplectic field theory, Math. Z. 248(1) (2004), 123-146.

[5] K. Cieliebak, T. Ekholm, J. Latschev and L. Ng, in preparation.

[6] K. Cieliebak and J. Latschev, The role of string topology in symplectic field theory, in 'New perspectives and challenges in symplectic field theory' (M. Abreu, F. Lalonde and L. Polterovich eds.), CRM Proc. 49 2009, 113-146.

[7] K. Cieliebak and K. Mohnke, Compactness for punctured holomorphic curves, J. Symplectic Geom. 3(4) (2006), 1-65.

[8] T. Ekholm, J. Etnyre and M. Sullivan, The contact homology of Legendrian submanifolds in $\mathbb{R}^{2 n+1}$, J. Differential Geom. 71(2) (2005), 177-305. 
[9] Y. Eliashberg, A. Givental and H. Hofer, Introduction to symplectic field theory, GAFA 2000 visions in mathematics special volume, part II, Birkhäuser, 560-673.

[10] C. Faber and R. Pandharipande, Hodge integrals and Gromov-Witten theory, Invent. Math. 139(1) (2000), 173-199.

[11] H. Hofer, K. Wysocki and E. Zehnder, Properties of pseudoholomorphic curves in symplectisations I: asymptotics, A. Inst. Henri Poincaré 13(3) (1996), 337-379.

[12] H. Hofer, K. Wysocki and E. Zehnder, Finite energy cylinders of small area, Ergodic Theory Dyn. Syst. 22(5) (2002), 1451-1486.

[13] S. Ivashkovich and V. Shevchishin, Reflection principle and J-complex curves with boundary on totally real immersions, Commun. Contemp. Math. 4(1) (2002), 65-106.

[14] C.-C. M. Liu, Moduli of J-holomorphic curves with lagrangian boundary conditions and open Gromov-Witten invariants for an $S^{1}$-equivariant pair, 2004, arXiv:math/0210257, v2.

[15] D. McDuff and D. Salamon, J-holomorphic curves and symplectic topology, AMS Colloquium Publications, 52, 2004, AMS, Providence.

[16] L. Ng, Framed knot contact homology, Duke Math. J. 141(2) (2008), 365-406.

[17] M. Schwarz, Cohomology operations from $S^{1}$-cobordisms in Floer homology $\mathrm{PhD}$ thesis, ETH Zürich, 1995.

[18] J. C. Sikorav, Some properties of holomorphic curves in almost complex manifolds in 'Holomorphic curves in symplectic geometry' (Audin, Lafontaine, eds.), 165-189, PM 117, Birkhäuser, Basel, 1994.

Mathematisches Institut, Ludwig-Maximilians-Universität, Theresienstr. 39, 80333 München, Germany

E-mail address: kai@math.lmu.de

Department of Mathematics, Uppsala University, Box 480, 75106 Uppsala, SWEDEN

E-mail address: tobias@math.uu.se

Departement Mathematik, ETH ZÜrich, RÄmistrasse 101, 8092 Zürich,

SWITZERLAND

E-mail address: janko@math.ethz.ch

Received 4/13/2009, accepted 1/19/2010

TE was supported by the Göran Gustafsson Foundation for Research in Natural Sciences and Medicine.

KC was partially supported by DFG grant CI 45/2-3. 